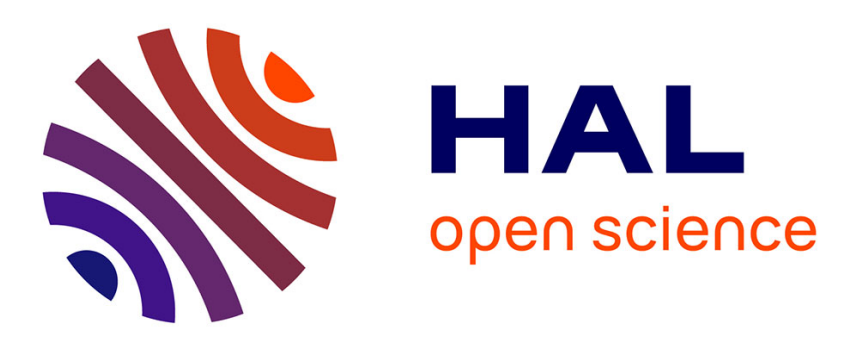

\title{
Estimating quadratic variation consistently in the presence of endogenous and diurnal measurement error
}

\author{
Ilze Kalnina, Oliver Linton
}

\section{To cite this version:}

Ilze Kalnina, Oliver Linton. Estimating quadratic variation consistently in the presence of endogenous and diurnal measurement error. Econometrics, 2008, 147 (1), pp.47. 10.1016/j.jeconom.2008.09.016 . hal-00533604

\section{HAL Id: hal-00533604 \\ https://hal.science/hal-00533604}

Submitted on 8 Nov 2010

HAL is a multi-disciplinary open access archive for the deposit and dissemination of scientific research documents, whether they are published or not. The documents may come from teaching and research institutions in France or abroad, or from public or private research centers.
L'archive ouverte pluridisciplinaire HAL, est destinée au dépôt et à la diffusion de documents scientifiques de niveau recherche, publiés ou non, émanant des établissements d'enseignement et de recherche français ou étrangers, des laboratoires publics ou privés. 


\section{Accepted Manuscript}

Estimating quadratic variation consistently in the presence of

endogenous and diurnal measurement error

Ilze Kalnina, Oliver Linton

PII:

S0304-4076(08)00123-1

DOI:

10.1016/j.jeconom.2008.09.016

Reference:

ECONOM 3079

To appear in: Journal of Econometrics

Please cite this article as: Kalnina, I., Linton, O., Estimating quadratic variation consistently in the presence of endogenous and diurnal measurement error. Journal of Econometrics (2008), doi:10.1016/j.jeconom.2008.09.016

This is a PDF file of an unedited manuscript that has been accepted for publication. As a service to our customers we are providing this early version of the manuscript. The manuscript will undergo copyediting, typesetting, and review of the resulting proof before it is published in its final form. Please note that during the production process errors may be discovered which could affect the content, and all legal disclaimers that apply to the journal pertain. 


\title{
Estimating Quadratic Variation Consistently in the presence of Endogenous and Diurnal Measurement Error*
}

\author{
Ilze Kalnina ${ }^{\dagger}$ and Oliver Linton ${ }^{\ddagger}$ \\ The London School of Economics
}

June 7, 2008

\begin{abstract}
We propose an econometric model that captures the effects of market microstructure on a latent price process. In particular, we allow for correlation between the measurement error and the return process and we allow the measurement error process to have a diurnal heteroskedasticity. We propose a modification of the TSRV estimator of quadratic variation. We show that this estimator is consistent, with a rate of convergence that depends on the size of the measurement error, but is no worse than $n^{-1 / 6}$. We investigate in simulation experiments the finite sample performance of various proposed implementations.
\end{abstract}

KEYWORDS: Endogenous noise; Market Microstructure; Realised Volatility; Semimartingale

JEL Classification: C12

\footnotetext{
*We would like to thank Jiti Gao, Dimitri Vayanos, Neil Shephard, Andrew Patton, Mathieu Rosenbaum, Yacine Aït-Sahalia, and two referees for helpful comments, as well as seminar participants at Perth, Alice Springs ESAM, Shanghai, and Yale. This research was supported by the ESRC and the Leverhulme foundation.

${ }^{\dagger}$ Department of Economics, London School of Economics, Houghton Street, London WC2A 2AE, United Kingdom. E-mail address: i.kalnina@lse.ac.uk

${ }^{\ddagger}$ Department of Economics, London School of Economics, Houghton Street, London WC2A 2AE, United Kingdom. E-mail address: o.linton@lse.ac.uk.
} 


\section{Introduction}

It has been widely recognized that using very high frequency data requires taking into account the effect of market microstructure (MS) noise. We are interested in the estimation of the quadratic variation of a latent price in the case where the observed log-price $Y$ is a sum of the latent log-price $X$ that evolves in continuous time and an error $u$ that captures the effect of MS noise.

There is by now a large literature that uses realized variance as a nonparametric measure of volatility. The justification is that in the absence of market microstructure noise it is a consistent estimator of the quadratic variation as the time between observations goes to zero. For a literature review, see Barndorff-Nielsen and Shephard (2007). In practice, ignoring microstructure noise seems to work well for frequencies below 10 minutes. For higher frequencies realized variance is not robust, as has been evidenced in the so-called 'volatility signature plots', see, e.g. Andersen et. al. (2000).

The additive measurement error model where $u$ is independent of $X$ and i.i.d. over time was first introduced by Zhou (1996). The usual realized volatility estimator is inconsistent under this assumption. The first consistent estimator of quadratic variation of the latent price in the presence of MS noise was proposed by Zhang, Mykland, and Ait-Sahalia (2005a) who introduced the Two Scales Realized Volatility (TSRV) estimator, and derived the appropriate central limit theory. TSRV estimates the quadratic variation using a combination of realized variances computed on two different time scales, performing an additive bias correction. It has a rate of convergence $n^{-1 / 6}$. Zhang (2004) introduced the more complicated Multiple Scales Realized Volatility (MSRV) estimator that combines multiple $\left(\sim n^{1 / 2}\right)$ time scales, which has a convergence rate of $n^{-1 / 4}$. This is known to be the optimal rate for this problem. Both papers assumed that the MS noise was i.i.d. and independent of the latent price. This assumption, according to an empirical analysis of Hansen and Lunde (2006), "seems to be reasonable when intraday returns are sampled every 15 ticks or so". Further studies have tried to relax this assumption to allow modelling of even higher frequency returns. Aït-Sahalia, Mykland and Zhang (2006a) modify TSRV and MSRV estimators and achieve consistency in the presence of serially correlated MS noise. Another class of consistent estimators of the quadratic variation was proposed by Barndorff-Nielsen, Hansen, Lunde, and Shephard (2006). They introduce realized kernels, a general class of estimators that extends the unbiased but inconsistent estimator of Zhou (1996), and is based on a general weighting of realized autocovariances as well as realized variances. They show that realized kernels can be designed to be consistent and derive the central limit theory. They show that for particular choices of weight functions they can be asymptotically equivalent to TSRV and MSRV estimators, or even more efficient. Apart from the benchmark setup where the noise is i.i.d. and independent from the latent price Barndorff-Nielsen et. al. (2006) have two additional sections, one allowing for $\mathrm{AR}(1)$ structure in the noise, another with an additional 
endogenous term albeit one that is asymptotically degenerate.

We generalize the standard additive noise model (where the noise is i.i.d. and independent from the latent price) in three directions. The first generalization is allowing for (asymptotically nondegenerate) correlation between MS noise and the latent returns. This is motivated by a paper of Hansen and Lunde (2006), where, for very high frequencies: "the key result is the overwhelming evidence against the independent noise assumption. This finding is quite robust to the choice of sampling method (calendar-time or tick-time) and the type of price data (transaction prices or quotation prices)". ${ }^{1}$

Another generalization concerns the magnitude of the MS noise. All of the papers above, like most of related literature, assume that the variance of the MS noise is constant and does not change depending on the time interval between trades. We call this a large noise assumption. We explicitly model the magnitude of the MS noise via a parameter $\alpha$, where the $\alpha=0$ case corresponds to the benchmark case of large noise. We allow also $\alpha>0$ in which case the noise is "small" and specifically the variance of the noise shrinks to zero with the sample size $n$. The rate of convergence of our estimator depends on the magnitude of the noise, and can be from $n^{-1 / 6}$ to $n^{-1 / 3}$, where $n^{-1 / 6}$ is the rate of convergence corresponding to the "big" noise case when $\alpha=0$.

How could the size of the noise "depend" on the sample size? We give a fuller discussion of this issue below, but we note here two arguments. First, there is a negative relationship between the bidask spread (an important component of the MS noise for transaction data) and a number of (other) liquidity measures, including number of transactions during the day. This negative relationship is a stylized fact from the market microstructure literature. See, for example, Copeland and Galai (1983) and McInish and Wood (1992). Also, Awartani, Corradi and Distaso (2004) write that "an alternative model of economic interest [to the standard additive noise model] would be one in which the microstructure noise variance is positively correlated with the time interval". This is in principle a testable hypothesis. Using Dow Jones Industrial Average data, the authors test for and reject the hypothesis of constant variance of the MS noise across frequencies.

The third feature of our model is that we allow the MS noise to exhibit diurnal heteroscedasticity. This is motivated by the stylized fact in market microstructure literature that intradaily spreads and intradaily stock price volatility are described typically by a U-shape (or reverse J-shape). See Andersen and Bollerslev (1997), Gerety and Mulherin (1994), Harris (1986), Kleidon and Werner (1996), Lockwood and Linn (1990), and McInish and Wood (1992). Allowing for diurnal heteroscedasticity

\footnotetext{
${ }^{1}$ By "independent noise" Hansen and Lunde (2006) mean the combination of the i.i.d. assumption and the assumption that the noise is independent from the latent price. Our paper proposes to relax the second assumption. As to the first assumption, we do not allow for serial correlation in the noise. At the same time, we only impose approximate stationarity compared to Hansen and Lunde (2006) since we allow for intraday heteroscedasticity of the noise.
} 
in our model has the effect that the original TSRV estimator may not be consistent because of end effects. In some cases, instead of estimating the quadratic variation, it would be estimating some function of the noise. We propose a modification of the TSRV estimator that is consistent, without introducing new parameters to be chosen. Our model is not meant to be definitive and can be generalized in a number of ways.

The structure of the paper is as follows. Section 2 introduces the model. Section 3 describes the estimator. Section 4 gives the main result and the intuition behind it. Section 5 investigates the numerical properties of the estimator in a set of simulation experiments. Section 6 illustrates the ideas with an empirical study of IBM transaction prices. Section 7 concludes. We use $\Longrightarrow$ to denote convergence in distribution.

\section{The Model}

Suppose that the latent $(\log )$ price process $\left\{X_{t}, t \in[0, T]\right\}$ is a Brownian semimartingale solving the stochastic differential equation

$$
d X_{t}=\mu_{t} d t+\sigma_{t} d W_{t}
$$

where $W_{t}$ is standard Brownian motion, $\mu_{t}$ is a locally bounded predictable drift function, and $\sigma_{t}$ a càdlàg volatility function; both are independent of the process $\left\{W_{t}, t \in[0, T]\right\}$. The (no leverage) assumption of $\left\{\sigma_{t}, \mu_{t}, t \in[0, T\}\right.$ being independent of $\left\{W_{t}, t \in[0, T]\right\}$, though reasonable for exchange rate data, is unrealistic for stock price data. However, it is frequently used and makes the theoretical analysis more tractable. The simulation results suggest that this assumption does not change the result. Furthermore, in many other contexts the presence of leverage does not affect the limiting distributions, see Barndorff-Nielsen and Shephard (2002).

The additive noise model says that the noisy price $Y$ is observed at times $t_{1}, \ldots, t_{n}$ on some fixed domain $[0, T]$

$$
Y_{t_{i}}=X_{t_{i}}+u_{t_{i}}
$$

where $u_{t_{i}}$ is a random variable representing measurement error. Without loss of much generality we are going to restrict attention to the case of equidistant observations with $T=1$. This type of model was first introduced by Zhou (1996) who assumed that $u_{t_{i}}$ is i.i.d. over $i$ and independent of $\left\{X_{t}, t \in[0,1]\right\}$. In this case the signal to noise ratio for returns decreases with sample size, i.e., $\operatorname{var}\left(\Delta X_{t_{i}}\right) / \operatorname{var}\left(\Delta u_{t_{i}}\right) \rightarrow 0$ as $n \rightarrow \infty$, and at a specific rate such that $\lim _{n \rightarrow \infty} n \operatorname{var}\left(\Delta X_{t_{i}}\right) / \operatorname{var}\left(\Delta u_{t_{i}}\right)<$ $\infty$, which implies inconsistency of realized volatility. We are going to modify the properties of the process $\left\{u_{t_{i}}\right\}$ and its relation to $\left\{X_{t}, t \in[0,1]\right\}$. 
We would like to capture the idea that the measurement error can be small. This can be addressed by adopting a model $u_{t_{i}}=\sigma_{\epsilon} \epsilon_{t_{i}}$, where $\epsilon_{t_{i}}$ is an i.i.d. sequence with mean zero and variance one, and $\sigma_{\epsilon}$ is a parameter such that $\sigma_{\epsilon} \rightarrow 0$. Many authors have found small $\sigma_{\epsilon}$ in practice. As usual one wants to make inferences about data drawn from the true probability measure of the data where both $n$ is finite and $\sigma_{\epsilon}>0$ by working with a limiting case that is more tractable. In this case there are a variety of limits that one could take. Bandi and Russell (2006a) for example calculate the exact MSE of the statistic of interest, and then in equation (24) implicitly take $\sigma_{\epsilon} \rightarrow 0$ followed by $n \rightarrow \infty$. We instead take the sound and well established practice in econometrics of taking pathwise limits, that is we let $\sigma_{\epsilon}=\sigma_{\epsilon}(n)$ and then let $n \rightarrow \infty$. Such a limit with "small" noise has been used before to derive Edgeworth approximations (Zhang et. al. 2005b), to calculate optimal sampling frequency of inconsistent estimator for $Q V_{x}$ (Zhang et al. 2005a, eqn. 53), to estimate $Q V_{x}$ consistently when $X$ follows a pure jump process and $Y$ is observed fully and continuously (Large (2007)), and to estimate $Q V_{x}$ consistently in a pure rounding model (Li and Mykland 2006, Rosenbaum 2007). An example from MS modelling literature in microeconomics is Back and Baruch (2004) who show the link between the two key papers in asymmetric information modelling, Glosten and Milgrom (1985) and Kyle (1985) using a limit with small noise. In particular, they consider a limit of Glosten and Milgrom (1985) as the arrival rate of trades explodes (so the number of trades in any interval goes to infinity) and order size (and hence incremental information per trade) goes to zero, thus reaching the Kyle (1985) model as a limit. We are also mindful not to preclude the case where $\sigma_{\epsilon}(n)$ is "large" i.e., (in our framework) does not vanish with $n$, and our parameterization below allows us to do that.

We next present our model. We assume that

$$
\begin{aligned}
u_{t_{i}} & =v_{t_{i}}+\varepsilon_{t_{i}} \\
v_{t_{i}} & =\delta \gamma_{n}\left(W_{t_{i}}-W_{t_{i-1}}\right) \\
\varepsilon_{t_{i}} & =m\left(t_{i}\right)+n^{-\alpha / 2} \omega\left(t_{i}\right) \epsilon_{t_{i}}, \alpha \in[0,1 / 2)
\end{aligned}
$$

with $\epsilon_{t_{i}}$ i.i.d. mean zero and variance one and independent of the Gaussian process $\left\{W_{t}, t \in[0,1]\right\}$ with $E\left|\epsilon_{t_{i}}\right|^{4+\eta}<\infty$ for some $\eta>0$. The functions $m$ and $\omega$ are differentiable, nonstochastic functions of time. They are unknown as are the constants $\delta$ and $\alpha$. The usual benchmark measurement error model with noise being i.i.d. and independent from the latent price has $\alpha=0, \gamma_{n}=0$ and $\omega($. and $m($.$) constant (see, e.g., Barndorff-Nielsen and Shephard 2002, Zhang et. al. 2005a, Bandi and$ Russell 2006b).

The process for the latent log-price is motivated by the fundamental theory of asset prices, which states that, in a frictionless market, log-prices must obey a semi-martingale; we are specializing to the Brownian semimartingale case (1). We want to model log-prices at very high frequency where frictions are important and observed prices do not follow a semimartingale. One way of partly 
reconciling the evidence in volatility signature plots of the price behavior in very high and moderate frequencies is to assume that observed prices can be decomposed as in (2). The first component $X$ is a semi-martingale with finite quadratic variation, while the second component $u$ is not a semimartingale and has infinite quadratic variation. In particular, the increments in $u$ are of larger magnitude than that of $X$, and this difference is the key in identifying the quadratic variation of $X$. We split the noise component $u$ into an independent term $\varepsilon$ that has been considered in the literature, and a 1-dependent endogenous part $v$, which is correlated with $X$ due to being driven by the same Brownian motion. At the same time, $v$ preserves the features of not being a semi-martingale and having infinite quadratic variation, the main motivation of the way $\varepsilon$ is modelled.

There are three key parts to our model: the correlation between $u$ and $X$, the relative magnitudes of $u$ and $X$, and the heterogeneity of $u$. We have $E\left[u_{t_{i}}\right]=m\left(t_{i}\right)$ and $\operatorname{var}\left[u_{t_{i}}\right]=\delta^{2} \gamma_{n}^{2}\left(t_{i}-t_{i-1}\right)+$ $2 n^{-\alpha} \sigma_{\epsilon}^{2}(i / n)$. To have the variance of both terms in $u$ equal, we set $\gamma_{n}^{2}=n^{1-\alpha}$. This seems like a reasonable restriction if both components are generated by the same mechanism. In this case, both of the measurement error terms are $O_{p}\left(n^{-\alpha}\right)$. In our model the signal to noise ratio of returns varies with sample size in a way depending on $\alpha$ so that only $\lim _{n \rightarrow \infty} n^{1-\alpha} \operatorname{var}\left(\Delta X_{t_{i}}\right) / \operatorname{var}\left(\Delta u_{t_{i}}\right)<\infty$. We exploit the fact that for consistency of the TSRV estimator, it is enough to assume that noise increments are of larger order of magnitude than the latent returns, and the usual stronger assumption $\lim _{n \rightarrow \infty} n \operatorname{var}\left(\Delta X_{t_{i}}\right) / \operatorname{var}\left(\Delta u_{t_{i}}\right)<\infty$ is not necessary.

The process $\varepsilon_{t_{i}}$ is a special case of the more general class of locally stationary processes of Dahlhaus (1997). The generalization to allowing time varying mean and variance in the measurement error allows one to capture diurnal variation in the measurement error process, which is likely to exist in calendar time. Nevertheless, the measurement error in prices is approximately stationary under our conditions, which seems reasonable.

The term $v$ in $u$ induces a correlation between latent returns and the change in the measurement error, which can be of either sign depending on $\delta$. Correlation between $u$ and $X$ is plausible due to rounding effects, price stickiness, asymmetric information, or other reasons [Bandi and Russell (2006c), Hansen and Lunde (2006), Diebold (2006)]. ${ }^{2}$ In the special case that $\sigma_{t}=\sigma$ and $\omega\left(t_{i}\right)=\omega$, we find

$$
\operatorname{corr}\left(\Delta X_{t_{i}}, \Delta u_{t_{i}}\right) \simeq \frac{\delta}{\sqrt{\left[2 \delta^{2}+2 \omega^{2}\right]}} .
$$

In this case, the range of correlation is limited, although it is quite wide - one can obtain up to a correlation of $\pm 1 / \sqrt{2}$ depending on the relative magnitudes of $\delta, \omega$.

\footnotetext{
${ }^{2}$ In a recent survey of measurement error in microeconometrics models, Bound, Brown, and Mathiowetz (2001) emphasize 'mean-reverting' measurement error that is correlated with the signal.
} 
An alternative model for endogenous noise has been developed by Barndorff-Nielsen, Hansen, Lunde, and Shephard (2006). In our notation, they have the endogenous noise part such that $\operatorname{var}\left(v_{t_{i}}\right)=O(1 / n)$, and an i.i.d., independent from $X$ part with $\operatorname{var}\left(\varepsilon_{t_{i}}\right)=O(1)$. They conclude robustness of their estimator to this type of endogeneity, with no change to the first order asymptotic properties compared to the case where $v_{t_{i}}=0$.

The focus of this paper is on estimating increments in quadratic variation of the latent price process ${ }^{3}$ but estimation of parameters of the MS noise in our model is also of interest. We acknowledge that not all the parameters of our model are identifiable. In particular, the endogeneity parameter may not be identified unless one knows something about the distribution of $\epsilon$ and in particular that it is not Gaussian. ${ }^{4}$ However, other parameters are identified. In Linton and Kalnina (2005) we provided a consistent estimator of $\alpha$, see also Section 6 here for empirical implementation and discussion. Estimating the function $\omega(\tau)$ would allow us to measure the diurnal variation of the MS noise. In the benchmark measurement error model this is a constant $\omega(\tau) \equiv \omega$ that can be estimated consistently by $\sum_{i=1}^{n-1}\left(Y_{t_{i+1}}-Y_{t_{i}}\right)^{2} / 2 n$ (Bandi and Russell (2006b), Barndorff-Nielsen et. al. (2006), Zhang et. al. (2005a)). In our model, instead of $n^{-1}$, the appropriate scaling is $n^{\alpha-1}$. Such an estimator would converge to $\delta^{2}+\int \omega^{2}(u) d u$. Hence, this estimator would converge asymptotically to the integrated variance of the MS noise. Following Kristensen (2006), in the special case $\delta=0$, we could also estimate $\omega(\cdot)$ at some fixed point $\tau$ using kernel smoothing,

$$
\widehat{\omega}^{2}(\tau)=\frac{1}{2 n^{1-\alpha}} \frac{\sum_{i=1}^{n} K_{h}\left(t_{i-1}-\tau\right)\left(\Delta Y_{t_{i-1}}\right)^{2}}{\sum_{i=1}^{n} K_{h}\left(t_{i-1}-\tau\right)\left(t_{i}-t_{i-1}\right)} .
$$

When the observations are equidistant, this simplifies to $\widehat{\omega}^{2}(\tau)=\sum_{i=1}^{n} K_{h}\left(t_{i-1}-\tau\right)\left(\Delta Y_{t_{i-1}}\right)^{2} / 2 n^{-\alpha}$. In the above, $h$ is a bandwidth that tends to zero asymptotically and $K_{h}()=.K(. / h) / h$, where $K($. is a kernel function satisfying some regularity conditions. If we also allow for endogeneity $(\delta \neq 0)$,

\footnotetext{
${ }^{3}$ There is a question about whether one should care about the latent price or the actual price. This has been raised elsewhere, see Zhang, Mykland, and Aït-Sahalia (2005). We stick with the usual practice here, acknowledging that the presence of correlation between the noise and efficient price makes this even more debatable, Aït-Sahalia, Mykland, and Zhang (2006b). Also, note that we are following the literature and estimating the quadratic variation of the latent log-price and not the latent price.

${ }^{4}$ Suppose that $X_{i+1}=X_{i}+(\sigma / \sqrt{n}) z_{i+1}$ and $Y_{i}=X_{i}+\rho z_{i}+\sigma_{\epsilon} \epsilon_{i}$, where $z_{i}$ is standard normal and $\varepsilon_{i}$ is i.i.d. with mean zero and variance one. Then $r_{i+1}=Y_{i+1}-Y_{i}=\left(\frac{\sigma}{\sqrt{n}}+\rho\right) z_{i+1}-\rho z_{i}+\sigma_{\epsilon} \epsilon_{i+1}-\sigma_{\epsilon} \epsilon_{i}$. We have $\operatorname{var}\left[r_{i+1}\right]=$ $2\left(\rho^{2}+\sigma_{\epsilon}^{2}\right)+\frac{2 \rho \sigma}{\sqrt{n}}+\frac{\sigma^{2}}{n}, \operatorname{cov}\left[r_{i+1}, r_{i}\right]=-\left(\rho^{2}+\sigma_{\epsilon}^{2}\right)-\frac{\rho \sigma}{\sqrt{n}}$, and $\operatorname{cov}\left[r_{i+j}, r_{i}\right]=0, j>1$. Therefore, from the covariogram we obtain $\sigma^{2}=n\left(\operatorname{var}\left[r_{i+1}\right]+2 \operatorname{cov}\left[r_{i+1}, r_{i}\right]\right)$ but we can only identify $\rho^{2}+\sigma_{\epsilon}^{2}$ not the two quantities separately. There are just two equations in two unknowns and if $\epsilon_{i}$ is also Gaussian, then there is no more information. If there is a non-Gaussian distribution one can identify $\rho$ using parametric restrictions. This is similar to the classical measurement error problem, Maddala, (1977, p 296).
} 
$\widehat{\omega}^{2}(\tau)$ estimates $\omega^{2}(\tau)$ plus a constant, and so we still see the pattern of diurnal variation. See Section 6 for implementation.

\section{Estimation}

We suppose that the parameter of interest is the quadratic variation of $X$ on $[0,1]$, denoted $Q V_{X}=$ $\int_{0}^{1} \sigma_{t}^{2} d t$. Let

$$
[Y, Y]^{n}=\sum_{i=1}^{n-1}\left(Y_{t_{i+1}}-Y_{t_{i}}\right)^{2}
$$

be the realized variation (often called realized volatility) of $Y$, and introduce a modified version of it (jittered RV) as follows,

$$
[Y, Y]^{\{n\}}=\frac{1}{2}\left(\sum_{i=1}^{n-K}\left(Y_{t_{i+1}}-Y_{t_{i}}\right)^{2}+\sum_{i=K}^{n-1}\left(Y_{t_{i+1}}-Y_{t_{i}}\right)^{2}\right) .
$$

This modification is useful for controlling the end effects that arise due to heteroscedasticity.

Our estimator of $Q V_{X}$ makes use of the same principles as the TSRV estimator in Zhang et. al. (2005a). We split the original sample of size $n$ into $K$ subsamples, with the $j^{\text {th }}$ subsample containing $n_{j}$ observations. Introduce a constant $\beta$ and $c$ such that $K=c n^{\beta}$. The dependence of $K$ on $n$ is suppressed in the sequel. For consistency we will need $\beta>1 / 2-\alpha$. The optimal choice of $\beta$ is discussed in the next section. By setting $\alpha=0$, we get the condition for consistency in Zhang et. al. (2005a), that $\beta>1 / 2 .^{5}$

Let $[Y, Y]^{n_{j}}$ denote the $j^{\text {th }}$ subsample estimator based on a $K$-spaced subsample of size $n_{j}$,

$$
[Y, Y]^{n_{j}}=\sum_{i=1}^{n_{j}-1}\left(Y_{t_{i K+j}}-Y_{t_{(i-1) K+j}}\right)^{2}, j=1, \ldots, K,
$$

and let

$$
[Y, Y]^{a v g}=\frac{1}{K} \sum_{j=1}^{K}[Y, Y]^{n_{j}}
$$

be the averaged subsample estimator. To simplify the notation, we assume that $n$ is divisible by $K$ and hence the number of data points is the same across subsamples, $n_{1}=n_{2}=\ldots=n_{K}=n / K$. Let $\bar{n}=n / K$.

\footnotetext{
${ }^{5}$ This condition is implicit in Zhang et al. (2005) in Theorem 1 (page 1400) where the rate of convergence is $\sqrt{K / \bar{n}}=c \sqrt{n^{2 \beta-1}}$.
} 
Define the adjusted TSRV estimator (jittered TSRV) as

$$
\widehat{Q V}_{X}=[Y, Y]^{a v g}-\left(\frac{\bar{n}}{n}\right)[Y, Y]^{\{n\}} .
$$

Compared to the TSRV estimator, this estimator does not involve any new parameters that would have to be chosen by the econometrician, so it is as easy to implement. The need to adjust the TSRV estimator arises from the fact that under our assumptions TSRV is not always consistent. The problem arises due to end-of-sample effects induced by heteroscedastic noise. For a simple example where the TSRV estimator is inconsistent, let us simplify the model to the framework of Zhang et. al. (2005a), and introduce only heteroscedasticity in the noise, the exact form of which is to be chosen below. Let us evaluate the asymptotic bias of TSRV estimator. ${ }^{6}$

$$
\begin{aligned}
& n^{1 / 6} E\left\{\widehat{Q V}_{X}^{T S R V}-Q V_{X}\right\} \\
= & n^{1 / 6}\left\{E[u, u]^{a v g}-\frac{\bar{n}}{n} E[u, u]^{n}\right\}+o(1) \\
= & c^{-1} n^{-1 / 2} \sum_{i=1}^{n-K}\left(\omega_{t_{i+K}}^{2} \epsilon_{t_{i+K}}^{2}+\omega_{t_{i}}^{2} \epsilon_{t_{i}}^{2}\right)-\left(c^{-1} n^{-1 / 2}-n^{-5 / 6}\right) \sum_{i=1}^{n-1}\left(\omega_{t_{i+1}}^{2} \epsilon_{t_{i+1}}^{2}+\omega_{t_{i}}^{2} \epsilon_{t_{i}}^{2}\right)+o(1) \\
= & n^{-5 / 6} \sum_{i=1}^{n-1}\left(\omega_{t_{i+1}}^{2} \epsilon_{t_{i+1}}^{2}+\omega_{t_{i}}^{2} \epsilon_{t_{i}}^{2}\right)-c^{-1} n^{-1 / 2}\left\{\sum_{i=2}^{K} \omega_{t_{i}}^{2} \epsilon_{t_{i}}^{2}+\sum_{i=n-K+1}^{n-1} \omega_{t_{i}}^{2} \epsilon_{t_{i}}^{2}\right\}+o(1) .
\end{aligned}
$$

We see that the first and last $K$ returns that are "ignored" by averaged subsampled realized volatility $[Y, Y]^{a v g} \sim[u, u]^{a v g}$ have to be off-set by a fraction of the noise of all returns, coming from $[Y, Y]^{n} \sim$ $[u, u]^{n}$. For this bias correction to work, the volatility of the microstructure noise in the morning and afternoon has to be "close" to the volatility of the noise during the day. A simple counter-example that is motivated by our empirical section 6.3 is a parabola on $[0,1], \omega^{2}(i / n)=a+\left(\frac{i}{n}-0.5\right)^{2} / 100$, where $a$ is any constant. In this case simple calculations give that TSRV estimator is inconsistent,

$$
n^{1 / 6} \mathrm{E}\left(\widehat{Q V}_{X}^{T S R V}-Q V_{X}\right)=-\frac{1}{300} n^{1 / 6}+o(1) .
$$

By contrast, jittered $R V,[Y, Y]^{\{n\}}$, mimics the structure of the volatility component that needs to be bias corrected for in $[Y, Y]^{\text {avg }}$, which is

$$
\frac{1}{\sqrt{n}} \sum_{i=1}^{n-K}\left(\omega_{t_{i+K}}^{2} \epsilon_{t_{i+K}}^{2}+\omega_{t_{i}}^{2} \epsilon_{t_{i}}^{2}\right)
$$

and so delivers a consistent estimator $\widehat{Q V}_{X}$.

\footnotetext{
${ }^{6}$ For the reader to be able to follow our calculations in the next few lines, she should use the exact definition of $\bar{n}, \bar{n}=\frac{n-K+1}{K}$ that Zhang et al. (2005) use. For all other purposes differences between our and their definition are negligible.
} 
We remark that (5) is an additive bias correction and there is a nonzero probability that $\widehat{Q V}_{X}<0$. One can ensure positivity by replacing $\widehat{Q V}_{X}$ by $\max \left\{\widehat{Q V}_{X}, 0\right\}$, but this is not very satisfactory. Note, however, that we usually have $\widehat{Q V}_{X}>\widehat{Q V}_{X}^{T S R V}$ (except for when first and last subsamples have all flat prices and so $\widehat{Q V}_{X}=\widehat{Q V}_{X}^{T S R V}$ ), so the probability that $\widehat{Q V}_{X}<0$ is lower than the probability that $\widehat{Q V}_{X}^{T S R V}<0$.

\section{Asymptotic Properties}

The expansion for $[Y, Y]^{a v g}$ and $[Y, Y]^{n}$ both contain terms due to the correlation between the measurement error and the latent returns. The main issues can be illustrated using the expansion of $[Y, Y]^{\text {avg }}$, conditional on the path of $\sigma_{t}$ :

$$
[Y, Y]^{a v g}=\underbrace{Q V_{X}}_{(a)}+\underbrace{\frac{\delta \gamma_{n}}{K} \int_{0}^{1} \sigma_{t} d t}_{(b)}+\underbrace{E[u, u]^{a v g}}_{(c)}+O(\underbrace{\overline{n^{-1 / 2}}}_{(d)}+\underbrace{\sqrt{\frac{\bar{n}}{K n^{2 \alpha}}}}_{(e)}) Z,
$$

where $Z \sim N(0,1)$, while the terms in curly braces are as follows: (a) the probability limit of $[X, X]^{a v g}$, which we aim to estimate; (b) the bias due to correlation between the latent returns and the measurement error; (c) the bias due to measurement error; (d) the variance due to discretization; (e) the variance due to measurement error.

Should we observe the latent price without measurement error, (a) and (d) would be the only terms. In this case, of course, it is better to use $[X, X]^{n}$, since that has an error of smaller order $n^{-1 / 2}$. In the presence of the measurement error, however, both $[Y, Y]^{\text {avg }}$ and $[Y, Y]^{n}$ are badly biased, the bias arising both from correlation between the latent returns and the measurement error, and from the variance of the measurement error. The largest term is (c), which satisfies

$$
E[u, u]^{a v g}=2 \bar{n} n^{-\alpha}\left(\int_{0}^{1} \omega^{2}(u) d u+\delta^{2}\right)+O\left(n^{-\alpha}+\bar{n}^{-1}\right)=O\left(\bar{n} n^{-\alpha}\right),
$$

i.e., it is of order $\bar{n} n^{-\alpha}$. So without further modifications, this is what $[Y, Y]^{\text {avg }}$ would be estimating. Should we be able to correct that, the next term would be $2\left(\delta \gamma_{n} / K\right) \int \sigma_{t} d t$ arising from $E[X, u]^{a v g}$. This second term is zero, however, if there is no correlation between the latent price and the MS noise, i.e., if $\delta=0$. Interestingly when we use the TSRV estimator for bias correction of $E[u, u]^{\text {avg }}$, we also cancel this second term.

The asymptotic distribution of our estimator arises as a combination of two effects, measurement error and discretization effect. After correcting for the bias due to the measurement error (terms 
like $b$ and $c$ in eqn. 6), we still have the variation due to the measurement error (term $e$ in eqn. 6 ). We can see that its contribution to the asymptotic distribution by observing how the estimator converges to the realized variance of the latent price $X$,

$$
\sqrt{\frac{K n^{2 \alpha}}{\bar{n}}}\left(\widehat{Q V}_{X}-[X, X]^{a v g}\right) \Longrightarrow N\left(0,8 \delta^{4}+16 \delta^{2} \int_{0}^{1} \omega^{2}(u) d u+8 \int_{0}^{1} \omega^{4}(u) d u\right),
$$

The rate of convergence arises from $\operatorname{var}[u, u]^{\text {avg }}=O\left(\bar{n} / K n^{2 \alpha}\right)$. Both parts of the noise $u$, which are $v$ and $\varepsilon$, contribute to the asymptotic variance. The first part of the asymptotic variance roughly arises from $\operatorname{var}[v, v]$, the second part from $\operatorname{var}[v, \varepsilon]$ (which is nonzero even though the correlation between both terms is zero), and the third part from $\operatorname{var}[\varepsilon, \varepsilon]$. If the measurement error is uncorrelated with the latent price, the first two terms disappear.

Should we observe the latent price without any error, we would still not know its quadratic variation due to observing the latent price only at discrete time intervals. This is another source of estimation error. From Theorem 3 in Zhang et. al. (2005a) we have

$$
\bar{n}^{1 / 2}\left([X, X]^{a v g}-Q V_{X}\right) \Longrightarrow M N\left(0, \frac{4}{3} \int_{0}^{1} \sigma_{t}^{4} d t\right),
$$

where $M N(0, S)$ denotes a mixed normal distribution with conditional variance $S$ independent of the underlying normal random variable.

The final result is a combination of the two results (7) and (8), as well as the fact that they are asymptotically independent. The fastest rate of convergence is achieved by choosing $K$ so that the variance from the discretization is of the same order as the variance arising from the MS noise, so set $\bar{n}^{-1 / 2}=\sqrt{\bar{n} / K n^{2 \alpha}}$. The resulting optimal magnitude of $K$ is such that $\beta=2(1-\alpha) / 3$. The rate of convergence with this rule is $\bar{n}^{-1 / 2}=n^{-1 / 6-\alpha / 3}$. The slowest rate of convergence is $n^{-1 / 6}$, and it corresponds to large MS noise case, $\alpha=0$. The fastest rate of convergence is $n^{-1 / 3}$, which corresponds to $\alpha=1 / 2$ case. If we pick a larger $\beta$ (and hence more subsamples $K$ ) than optimal, the rate of convergence in (7) increases, and the rate in (8) decreases and so dominates the final convergence result. In this case the final convergence is slower and only the first term due to discretization appears in the asymptotic variance (see (9)). Conversely, if we pick a smaller $\beta$ (and hence $K$ ) than optimal, we get a slower rate of convergence and only the second term in the asymptotic variance ("measurement error" in (9)), which is due to the MS noise.

We obtain the asymptotic distribution of $\widehat{Q V}_{X}$ in the following theorem

Theorem. Suppose that $\left\{X_{t}, t \in[0,1]\right\}$ is a Brownian semimartingale satisfying (1). Suppose that $\left\{\mu_{t}, t \in[0,1]\right\}$ and $\left\{\sigma_{t}, t \in[0,1]\right\}$ are measurable and càdlàg processes, independent of the process $\left\{W_{t}, t \in[0,1]\right\}$. Suppose further that the observed price arises as in (2) with $\alpha \in[0,1 / 2)$. Let the 
measurement error $u_{t_{i}}$ be generated by (3), with $\epsilon_{t_{i}}$ i.i.d. mean zero and variance one and independent of the Gaussian process $\left\{W_{t}, t \in[0,1]\right\}$ with $E\left|\epsilon_{t_{i}}\right|^{4+\eta}<\infty$ for some $\eta>0$. Then,

$$
\begin{gathered}
V(\sigma)^{-1 / 2} \bar{n}^{1 / 2}\left(\widehat{Q V}_{X}-Q V_{X}\right) \Longrightarrow N(0,1) \\
V(\sigma)=\underbrace{\frac{4}{3} \int_{0}^{1} \sigma_{t}^{4} d t}_{\text {discretization }}+\underbrace{c^{-3}\left(8 \delta^{4}+16 \delta^{2} \int_{0}^{1} \omega^{2}(u) d u+8 \int_{0}^{1} \omega^{4}(u) d u\right)}_{\text {measurement error }}>0 \text { a.s. }
\end{gathered}
$$

\section{REMARKS.}

1. The quantity $V(\sigma)$ collapses to the expression in Zhang et. al. (2005a) when $\omega($.$) is constant.$

2. If one could find a consistent estimator $\widehat{V}(\sigma)$ such that $\widehat{V}(\sigma)-V(\sigma)=o(1)$ a.s., then the above theorem can be strengthened along the lines of Barndorff-Nielsen and Shephard to a feasible CLT, i.e., $\widehat{V}(\sigma)^{-1 / 2} \bar{n}^{1 / 2}\left(\widehat{Q V}_{X}-Q V_{X}\right) \Longrightarrow N(0,1)$ from which one could obtain confidence intervals for $Q V_{X}$. Without assuming $\delta=0$ or constant $\omega($.$) , the procedure of Zhang et. al. (2005a), p. 1404,$ would work to estimate $V(\sigma)$.

3. The main statement of the theorem can also be written as

$$
n^{1 / 6+\alpha / 3}\left(\widehat{Q V}_{X}-Q V_{X}\right) \Longrightarrow M N(0, c V(\sigma)),
$$

where $V(\sigma)=V_{1}(\sigma)+c^{-3} V_{2}$, with $V_{1}(\sigma)$ being the discretization error, while $M N$ denotes a mixed normal distribution with conditional variance $c V(\sigma)$ independent of the underlying normal random variable. We can use this to find the value of $c$ that would minimize the conditional asymptotic variance, $c_{o p t}(\sigma)=\left(2 V_{2} / V_{1}(\sigma)\right)^{1 / 3}$, provided $V_{1}(\sigma)>0$, resulting in the asymptotic conditional variance $\left(3 / 2^{2 / 3}\right) V_{2}^{1 / 3} V_{1}^{2 / 3}(\sigma)$. If one has consistent estimators $\widehat{V}_{j}(\sigma)-V_{j}(\sigma)=o(1)$ a.s., $j=1,2$, then $\widehat{c}_{\text {opt }}(\sigma)=\left(2 \widehat{V}_{2}(\sigma) / \widehat{V}_{1}(\sigma)\right)^{1 / 3}$ is consistent in the sense that $\widehat{c}_{\text {opt }}(\sigma)-c_{\text {opt }}(\sigma)=o(1)$ a.s.

4. Suppose now that the measurement error is smaller than above and we have $\alpha \in[1 / 2,1)$ instead of $\alpha \in[0,1 / 2)$. Then, there is a consistency condition $\beta>1 / 3$ that becomes binding and therefore optimal $\beta$ allows the measurement error to converge faster than the discretization error. For $\beta=1 / 3+\Delta$ (where $\Delta$ small and positive) the rate of convergence is $\bar{n}^{-1 / 2}=n^{-(1-\beta) / 2}=n^{-1 / 3+\Delta / 2}$. Note that this is exactly the rate that occurs when there is no measurement error at all. So choose $\beta \in(1 / 3,1)$. The conclusion of the theorem becomes

$$
V(\sigma)^{-1 / 2} n^{(1-\beta) / 2}\left(\widehat{Q V}_{X}-Q V_{X}\right) \Longrightarrow N(0,1)
$$

where $V_{1}(\sigma)=(4 / 3) \int \sigma_{t}^{4} d t$. This can be shown by minor adjustments to the proofs.

5 . What if $\alpha \geq 1$ ? This means that $[u, u]$ is of the same or smaller magnitude than $[X, X]$. In the case $\alpha=1$ they are of the same order and identification breaks down. When $\alpha>1$, realized volatility 
of observed prices is a consistent estimator of quadratic variation of latent prices, as measurement error is of smaller order. This is an artificial case and does not seem to appear in the real data.

How can we put this analysis in context? A useful benchmark for evaluation of the asymptotic properties of nonparametric estimators is the performance of parametric estimators. Gloter and Jacod (2001) allow for the dependence of the variance of i.i.d. Gaussian measurement error $\rho_{n}$ on $n$ and establish the Local Asymptotic Normality (LAN) property of the likelihood, which is a precondition to asymptotic optimality of the MLE. For the special case $\rho_{n}=\rho$ they obtain a convergence rate $n^{-1 / 4}$, thus allowing one to conclude that the MSRV and realized kernels can achieve the fastest possible rate. They also show that the rate of convergence is $n^{-1 / 2}$ if $\rho_{n}$ goes to zero sufficiently fast, which is the rate when there is no measurement error at all. Our estimator has a rate $n^{-1 / 3+\Delta}$ when there is no measurement error, which is also the rate of convergence when the noise is sufficiently small. Also, Gloter and Jacod have that for "large" noise, the rate of convergence depends on the magnitude of the noise, similarly to our results. The rate of convergence and the threshold for the magnitude of the variance of the noise is different, though.

\section{Simulation study}

In this section we explore the behavior of the estimator (5) in finite samples. We simulate the Heston (1993) model:

$$
\begin{aligned}
d X_{t} & =\left(\mu_{t}-v_{t} / 2\right) d t+\sigma_{t} d W_{t} \\
d v_{t} & =\kappa\left(\theta-v_{t}\right) d t+\gamma v_{t}^{1 / 2} d B_{t},
\end{aligned}
$$

where $v_{t}=\sigma_{t}^{2}$, and $W_{t}, B_{t}$ are independent standard Brownian motions.

For the benchmark model, we take the parameters of Zhang et. al. (2005a): $\mu=0.05, \kappa=5$, $\theta=0.04, \gamma=0.5$. We set the length of the sample path to 23400 corresponding to the number of seconds in a business day, the time between observations corresponding to one second when a year is one unit, and the number of replications to be $100,000 .{ }^{7}$ We set $\alpha=0$. We choose the values of $\omega$ and $\delta$ so as to have a homoscedastic measurement error with variance equal to $0.0005^{2}$ (again from

\footnotetext{
${ }^{7}$ Note that in the theoretical part of the paper we had for brevity taken interval $[0,1]$. For the simulations we need the interval $[0,1 / 250]$. Suppose the parameter of interest is $\int_{0}^{\tau} \sigma_{t}^{2} d t$, the quadratic variation of $X$ on $[0, \tau]$. In that case the asymptotic conditional variance of the theorem becomes

$$
V(\sigma)=\frac{4}{3} \tau \int_{0}^{\tau} \sigma_{t}^{4} d t+c^{-3}\left(8 \tau^{2} \delta^{4}+16 \delta^{2} \int_{0}^{\tau} \omega^{2}(u) d u+8 \tau^{-1} \int_{0}^{\tau} \omega^{4}(u) d u\right) .
$$

This follows by simple adjustments in the proofs. We take $\tau=1 / 250$.
} 
Zhang et. al. (2005a)), and correlation between the latent returns and the measurement error equal to -0.1 . For this we use the identity

$$
\operatorname{corr}\left(\Delta X_{t_{i}}, \Delta u_{t_{i}}\right)=\frac{E(\sigma)}{\sqrt{2 E\left(\sigma^{2}\right)}} \frac{\delta}{\sqrt{\delta^{2}+\omega^{2}}}
$$

and the fact that for our volatility we have $E(\sigma)=\theta$, $\operatorname{var}(\sigma)=\theta \gamma^{2} / 2 \kappa$. We set $\beta=2(1-\alpha) / 3$. Figure 1 shows the common volatility path for all simulations.

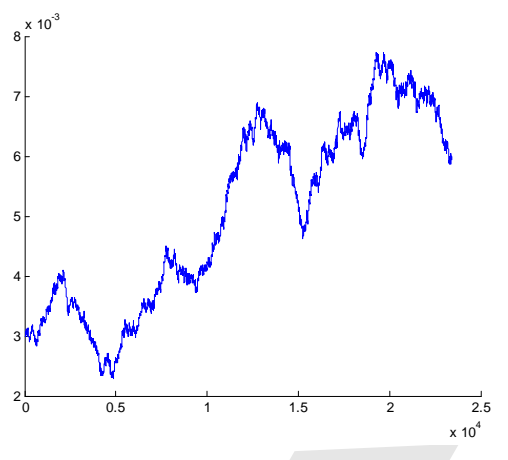

Figure 1. The common volatility path for all simulations.

First, we construct different models to see the effect of varying $\alpha$ and the number of observations within a day. We take the values of $\delta$ and $\omega$ that arise from the benchmark model, and then do simulations for the following combinations of $\alpha$ and $n$. When interpreting the results, we should also take into account that both of these parameters change the size of the variance of the measurement error. We measure the proximity of the finite sample distribution to the asymptotic distribution by the percentage errors of the interquartile range of $\bar{n}^{1 / 2}\left(\widehat{Q V}_{X}-Q V_{X}\right)$ compared to $1.3 \sqrt{V}$, the value predicted by the distribution theory. We note that this is not the same as the MSE or variance of the estimator: it can be that a very efficient estimator can be poorly approximated by its limiting distribution and vice versa. This measure is easiest to interpret if we work with a fixed variance, i.e., when we condition on the volatility path. Hence, we simulate the volatility path for the largest number of observations, 23400, and perform all simulations using this one sample path of volatility. The last parameter to choose is $K$, the number of subsamples. This is the only parameter that an econometrician has to choose in practice. We examine four different values as follows (the expressions are all rounded to the closest integer):

Table 1. Choices of $K$

\begin{tabular}{l|l|l}
\hline \hline$\left(2 V_{2} / V_{1}\right)^{1 / 3} n^{\frac{2}{3}(1-\alpha)}$ & asymptotically optimal rate and $c$ & Tables 2 and 3 \\
$n^{\frac{2}{3}(1-\alpha)}$ & variation of above & Tables 4 and 5 \\
$n^{\frac{2}{3}}$ & variation of above & Table 6 \\
$\left(\frac{3 R V^{2}}{2 R Q}\right)^{1 / 3} n^{1 / 3}$ & Bandi and Russell (2006a, eq. 24) & Table 7 \\
\hline \hline
\end{tabular}


Table 2 contains the interquartile range errors (IQRs), in per cent, with the asymptotically optimal rate and constant (in terms of minimizing asymptotic mean squared error) for $K$. That is, we use $K=\left(2 V_{2} / V_{1}\right)^{1 / 3} n^{2(1-\alpha) / 3}$, rounded to the nearest integer, where $V_{1}$ and $V_{2}$ are discretization and measurement errors from (9). Table 3 contains the values of $K$.

***Tables 2 -8 here***

First of all, for small values of $\alpha$, the percentage errors decrease with $n$ as predicted by the theory. However, we do see some large errors, and from the values of $K$ in Table 3 we can guess this is due to the asymptotically optimal rule selecting very low $c_{\text {opt }}$. In fact, for the volatility path used here, $c_{\text {opt }}=\left(2 V_{2} / V_{1}\right)^{1 / 3}=0.0242$. Hence, another experiment we consider is an arbitrary choice $c=1$. The next two tables (Table 4 and 5) contain the percentage errors and values of $K$ that result from using $K=n^{2(1-\alpha) / 3}$.

The performance of this choice is much better. We can see from Table 4 that for small values of $\alpha$, the asymptotic approximation improves with sample size. The sign of the error changes as $\alpha$ increases for given $n$, meaning that the actual IQR is below that predicted by the asymptotic distribution for small $\alpha$ and small $n$ but this changes into the actual IQR being above the asymptotic prediction.

Another variant that does not include the unobservable $\alpha$ would be to use $K=n^{2 / 3}$.

Finally, we consider a method proposed by Bandi and Russell (2006a), which requires some discussion. They establish the exact mean squared error of TSRV under the assumptions of the independent additive noise model, and in addition they assume asymptotically constant volatility, i.e., $\int_{t_{i-1}}^{t_{i}} \sigma_{u}^{2} d u=\int_{0}^{1} \sigma_{u}^{2} d u / n$ for each $i$, as well as $E\left(\epsilon^{4}\right)=3 E^{2}\left(\epsilon^{2}\right)$. Two assumptions are not satisfied in our simulation setup, the independence between the noise and the latent returns, as well as the assumption $\int_{t_{i-1}}^{t_{i}} \sigma_{u}^{2} d u=\int_{0}^{1} \sigma_{u}^{2} d u / n$ for each $i$ (see Figure 1). Therefore, this should be considered as another ad hoc selection method in our simulation setup. We note that this bandwidth choice results in an inconsistent estimator in our framework and in the framework of ZMA (2005a) (i.e., when $\alpha=0, \beta>1 / 2$ is required for consistency). Note that the choice $K^{B R}$ was derived for $\widehat{Q V}^{T S R V}$ without jittering, but this end-of-sample adjustment, though theoretically crucial, is negligible in simulations and, as we will see in the next section, also in real data. Table 7 contains the IQR percentage errors and values of $K$ that result from using $K^{B R}=\left(3 R V^{2} / 2 R Q\right)^{1 / 3} n^{1 / 3}$, where $R V$ is the realized variance, $R V=\sum\left(\Delta Y_{\text {low }}\right)^{2}$ and $R Q$ is the realized quarticity, $R Q=\frac{S}{3} \sum\left(\Delta Y_{\text {low }}\right)^{4}$. Here, $Y_{\text {low }}$ is low frequency ( 15 minute) returns, which gives $S=24$ to be the number of low frequency observations during one day.

We see that the IQR errors of this choice get worse with sample size for small $\alpha$, which reflects the inconsistency predicted by the theory. On the other hand the errors are small and improve with $n$ for 
large $\alpha$, i.e., when the noise is small. The performance is generally better than with asymptotically optimal $K$, except for cases that have both large $n$ and small $\alpha$, including the case $\alpha=0$ usually considered in the literature. We notice that $K^{B R}$ rule gives better results than the asymptotically optimal rule when it chooses a larger $K$, which is in most cases, but not all. In comparison to rules $K=n^{2(1-\alpha) / 3}$ and $K=n^{2 / 3}$ (Tables 4 and 6, respectively), the performance of this choice is still disappointing, especially for small $\alpha$. We conclude that in this setting the $K^{B R}$ rule is not always the best choice according to our criterion.

It has been noted elsewhere that the asymptotic approximation can perform poorly, see Gonçalves and Meddahi (2005) and Aït-Sahalia, Zhang and Mykland (2005a).

From Tables 2, 4, and 6 we see that magnitude of noise does not affect the quality of the asymptotic approximation. Although we see the interquartile range error having some relationship with $\alpha$ in Table 4 and especially Table 2, this is purely driven by changes in $K$. This is evidenced by Table 6 where the rule for $K$ does not depend on $\alpha$ and the respective error is close to constant for the same number of observations and different $\alpha$. Another conclusion here is that a good rule for $K$ does not necessarily have to depend on $\alpha$, which is convenient for practical purposes.

In a second set of experiments we investigate the effect of varying $\omega$, which controls the variance of the second part of the measurement error, for the largest sample size. Denoting by $\omega_{b}^{2}$ the value of $\omega^{2}$ in the benchmark model, we construct models with $\omega^{2}=\omega_{b}^{2}, 4 \omega_{b}^{2}, 8 \omega_{b}^{2}, 10 \omega_{b}^{2}$, and $20 \omega_{b}^{2}$. The corresponding interquartile errors are $0.96 \%, 1.26 \%, 1.93 \%, 2.29 \%$, and $4.64 \%$.

In a third set of experiments we investigate the effect of varying $\delta$, which controls the size of the correlation of the latent returns and measurement error. Denoting by $\delta_{b}^{2}$ the value of $\delta^{2}$ in the benchmark model, we construct models with $\delta^{2}$ being from $0.01 \times \delta^{2}$ to $20 \times \delta^{2}$. The exact values of $\delta^{2}$, as well as corresponding correlation between returns and increments of the noise, and the resulting interquartile errors are reported in Table 8 . We can see that when the number of observations is 23400 , there is no strong effect from the correlation of the latent returns and measurement error on the approximation of the asymptotic interquartile range of the estimator.

\section{Empirical analysis}

To illustrate the above ideas, we perform a small empirical analysis. We discuss estimation of $\alpha$, $\omega($.$) , and the quadratic variation of the latent price. The endogeneity parameter \delta$ is unfortunately nonparametrically unidentified and so cannot be estimated. Its sole purpose is in allowing for flexible size and sign of endogeneity, with respect to which our estimator of quadratic variation is robust.

Figure 5 in the appendix shows the volatility signature of the data we use, which is IBM transaction data, year 2005. The plot indicates that market microstructure noise is prevalent at the 
frequencies of $10-15$ minutes and higher. Since the volatility signature plot does not become negative, one cannot find evidence of endogeneity using the method of HL (2006). As pointed out already by $\mathrm{HL}(2006)$, this does not mean there is no endogeneity.

\subsection{The Data}

We use IBM transactions data for the whole year 2005. We employ the data cleaning procedure as in HL (2006), main paper and rejoinder. First, we use transactions from NYSE exchange only as this is the main exchange for IBM. Second, we use only transactions from 9:30AM to 4:00PM. Third, for transactions with the same time stamp, we use the average price. Fourth, we remove outliers as follows. If the price is too much above the ask price or too much below the bid, we remove it. Too high means more than spread above the ask, and too low means more than spread below the bid. Fifth, we remove days with less than 5 hours of trading (there were none). For discussion of the advantages of this procedure see HL (2006). The mean number of transactions per day in our cleaned data set is 4,484 (for comparison, there are 4,680 intervals of 5 seconds in the 6.5 hours between 9:30 and 16:00).

\subsection{Estimation of $\alpha$}

The parameter that governs the magnitude of the microstructure noise, $\alpha$, can be consistently estimated. Recall that the leading term of realized volatility $[Y, Y]^{n}$ is $[u, u]^{n}$ i.e.,

$$
\begin{aligned}
{[Y, Y]^{n} } & =\sum_{i=1}^{n-1}\left(u_{t_{i+1}}-u_{t_{i}}\right)^{2}+o_{p}\left(n^{1-\alpha}\right) \\
& =n^{-\alpha} \sum_{i=1}^{n-1}\left(\omega_{t_{i+1}} \epsilon_{t_{i+1}}-\omega_{t_{i}} \epsilon_{t_{i}}+\delta \sqrt{n}\left(W_{t_{i+1}}-W_{t_{i}}\right)\right)^{2}+o_{p}\left(n^{1-\alpha}\right) \\
& =n^{1-\alpha} c+o_{p}\left(n^{1-\alpha}\right)
\end{aligned}
$$

for some positive constant $c$. It follows that

$$
\log \left([Y, Y]^{n} / n\right)=-\alpha \log n+\log c+o_{p}(\log n) .
$$

We therefore estimate $\alpha$ by

$$
\widehat{\alpha}=-\frac{\log \left([Y, Y]^{n} / n\right)}{\log (n)},
$$

see Linton and Kalnina (2007).

Although this is a consistent estimator for $\alpha$, it has a bias that decays slowly. To reduce the bias, we estimate $\alpha$ over windows of 60 days instead of 1 day, i.e., we take our fixed interval $[0,1]$ to 
represent 3 months instead of 1 day. Figure 2 shows the estimates over the whole year 2005 where we roll the 60 day window by 1 day. We see that $\widehat{\alpha}$ varies between 0.64 and 0.7 with an average value of 0.67 .

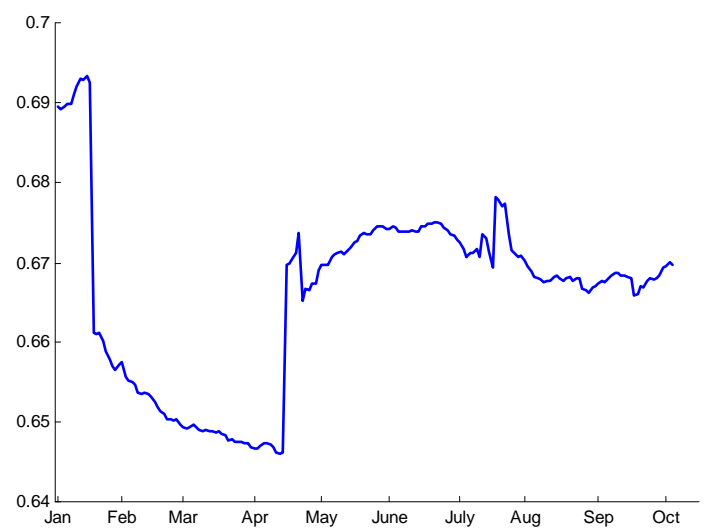

Figure 2. Estimated $\alpha$ over a rolling window of 60 days (approx. 3 months). $\mathrm{X}$ axis shows the date of the first day in the window.

Although this is a consistent estimator for $\alpha$, it is not precise enough to give a consistent estimator of $n^{\alpha}$. As a consequence, this estimator cannot be used for consistent inference for $\widehat{Q V}_{x}$. In Linton and Kalnina (2005) we provide a sharper bias adjusted version of $\widehat{\alpha}, \widehat{\alpha}^{\text {adj }}$, but the adjusted estimator is not feasible as it requires knowledge of $\omega(\tau)$. This last parameter can only be consistently estimated if $\alpha=0$ and $\delta=0$. The lack of precision in $\widehat{\alpha}$ also prevents us from developing a test of the null hypothesis $\alpha=0$. Therefore, the deviations of $\widehat{\alpha}$ we see in Figure 2 provide only a heuristic evidence that the true $\alpha$ is positive.

\subsection{Estimation of Scedastic function $\omega($.}

Now we estimate the function $\omega(\tau)$ that allows us to measure the diurnal variation of the MS noise. In the benchmark measurement error model this is a constant $\omega(\tau) \equiv \omega$ that can be estimated consistently by $\sum_{i=1}^{n-1}\left(Y_{t_{i+1}}-Y_{t_{i}}\right)^{2} / 2 n$ (Bandi and Russell (2006c), Barndorff-Nielsen et al. (2006), Zhang et al. (2005a)). In the special case $\alpha=0$ and $\delta=0$ this estimator would converge asymptotically to the integrated variance of the MS noise, $\int \omega^{2}(\tau) d \tau$. We can estimate the function $\omega^{2}($.$) at$ a specific point $\tau$ using a simple generalization of the approach of Kristensen (2006) to the case with market microstructure noise. For equidistant observations, the estimator is

$$
\widehat{\omega}^{2}(\tau)=\frac{\sum_{i=1}^{n} K_{h}\left(t_{i-1}-\tau\right)\left(\Delta Y_{t_{i-1}}\right)^{2}}{2 n^{-\alpha}} .
$$


We pick a random day, say 77th, which corresponds to 22nd of April. Assume $\alpha=0$ and $\delta=0$ and note that if these assumptions are not true, the level will be incorrect, while the diurnal variation will still be correct. Figure $3 \mathrm{~b}$ shows the estimated function $\widehat{\omega}^{2}(\tau)$ using calendar time with 30 seconds frequency. We see that the variance of MS noise is far from being constant, and is closer to U-shape. Higher $\widehat{\omega}^{2}(\tau)$ at the beginning of the day and low values around 13:00 are displayed by virtually all days in 2005, while higher values of $\widehat{\omega}^{2}(\tau)$ at the end of the day are less common. Hence, overall, we confirm the findings of the empirical market microstructure literature that the intraday patterns are of $\mathrm{U}$ or reverse $\mathrm{J}$ shape (see references in the introduction).

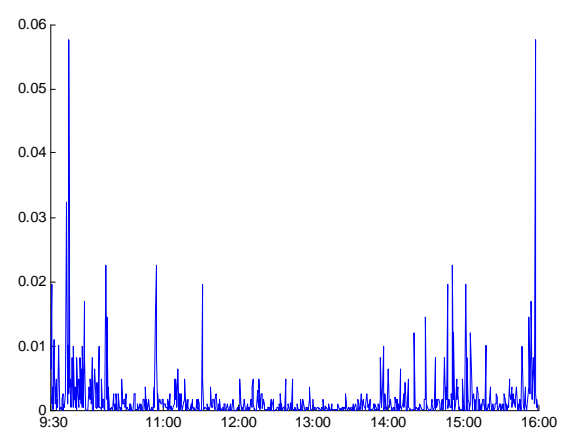

(a) Squared returns

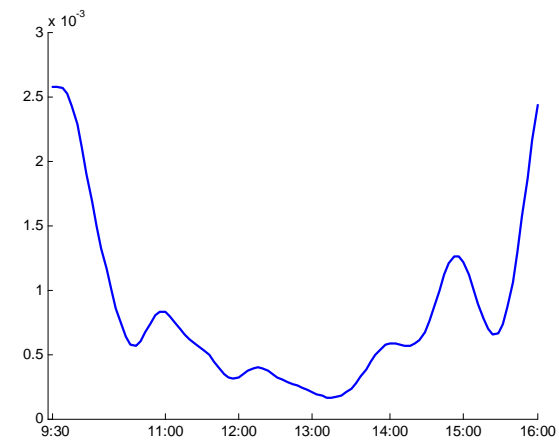

(b) Estimated function $\omega^{2}($.

Figure 3. IBM transactions data, 22nd of April 2005.

\subsection{Estimation of Quadratic Variation}

Our theory predicts that original $T S R V$ estimator is asymptotically as good as our jittered version if intraday volatility pattern is "close enough" to constant volatility. Visual inspection of the estimated volatilities in the previous section suggest that there is some deviation from constant volatility, so one might call for adjustment to the TSRV estimator. How important is this adjustment in practice?

We check empirically the effect of jittering on daily point estimates of quadratic variation using IBM data in 2005. Figure 4a shows a plot of relative differences

$$
\frac{\widehat{Q V}_{X}-\widehat{Q V}_{X}^{T S R V}}{\widehat{Q V}_{X}^{T S R V}}
$$

for every day in 2005 where we use tick time sampling (with 1-tick and $K=n^{2 / 3}$ ). The plot for 5 minute calendar time sampling (CTS) is very similar. The mean of these relative differences over all days is 0.0009 . Figure $4 \mathrm{~b}$ shows means of this relative difference for CTS, across different 
frequencies. ${ }^{8}$ We see that, on average, for high frequencies, jittering makes very little difference. For lower frequencies the change is more visible. This arises from the fact that the jittering changes the $T S R V$ estimator on two subsamples only (see eqn. 12). The more subsamples there are, the less important our adjustment (this can also be achieved for any fixed frequency by using larger number of subsamples than our choice $\left.K=n^{2 / 3}\right)$.

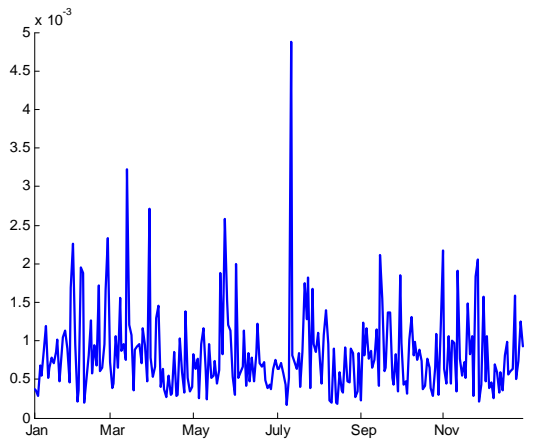

(a) daily differences, 1-tick sampling

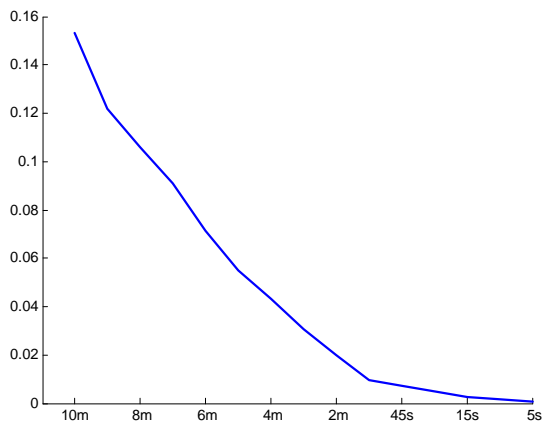

(b) average daily differences, CTS

Figure 4. What is the relative difference $\frac{\widehat{Q V}_{X}-\widehat{Q V}_{X}^{T S R V}}{\widehat{Q V}_{X}^{T S R V}}$ from our adjustment to the TSRV estimator?

Another important observation is that jittering always increases the value of QV estimates, since we can write

$$
\widehat{Q V}_{X}^{T S R V}=\widehat{Q V}_{X}+\frac{1}{2}\left(\sum_{i=1}^{K-1}\left(Y_{t_{i+1}}-Y_{t_{i}}\right)^{2}+\sum_{i=n-K+1}^{n}\left(Y_{t_{i+1}}-Y_{t_{i}}\right)^{2}\right)>\widehat{Q V}_{X} .
$$

The more there is variation in the beginning of the day and the end of the day, the larger is the adjustment. This implies that jittering partly alleviates the problem that the usual TSRV estimator can sometimes become negative. With our data set, the only negative value (though very small) we saw was on February 28 when we calculated TSRV estimator with 10 minutes CTS frequency. The jittered version was positive.

We conclude that for most applications our estimator is very close to the TSRV estimator, and so for practical applications plain $T S R V$ estimator can be used, without adjustment for heteroscedastic market microstructure noise. As a result, as far as point estimates are concerned, the existing empirical studies of $T S R V$ estimator are still valid in our theoretical framework. See, for example, investigations of forecasting performance in Aït-Sahalia and Mancini (2006), Andersen, Bollerslev, and Meddahi (2006), Bandi, Russell, and Yang (2007), and Ghysels and Sinko (2006).

\footnotetext{
${ }^{8}$ This average excludes October 27. On this day our estimator, when calculated on fequencies above 7 minutes, became several times bigger than TSRV estimator.
} 


\section{Conclusions and Extensions}

In this paper we showed that the TSRV estimator is consistent for the quadratic variation of the latent $(\log )$ price process when the measurement error is correlated with the latent price, although some adjustment is necessary when the measurement error is heteroscedastic. We also showed how the rate of convergence of the estimator depends on the magnitude of the measurement error.

Inference for TSRV estimator is robust to endogeneity of the measurement error. Provided the suggested adjustment to the estimator is implemented to preserve consistency, inference is also robust to heteroscedasticity of the noise. However, since the rate of convergence depends on the magnitude of the noise, inference is not robust to possible deviations from assumptions about this magnitude. We plan to investigate this question further.

Other examples where inference question needs to be solved include autocorrelation in measurement error (as in Ait-Sahalia, Mykland, and Zhang, 2006a), or other generalizations to the independent additive error model (Li and Mykland 2007). Gonçalves and Meddahi (2005) have recently proposed a bootstrap methodology for conducting inference under the assumption of no noise and shown that it has good small sample performance in their model. Zhang, Mykland, and Aït-Sahalia (2005b) have developed Edgeworth expansions for the TSRV estimator, and it would be very interesting to use this for analysis of inference using bootstrap. The results we have presented may be generalized to cover MSRV estimators and to allow for serial correlation in the error terms, although in both cases the notation becomes very complicated.

\section{A Appendix}

We assume for simplicity that $\mu \equiv 0$ in the sequel. Drift is not important in high frequencies as it is of order $d t$, while the diffusion term is of order $\sqrt{d t}$ (see, for example Aït-Sahalia et al.(2006)). With the assumptions of Theorem, the same method as in the proof can be applied to the drift, yielding the conclusion that it is not important statistically.

Proof of Theorem. We will rely on the first and second moment calculations of $[X, u]^{\{n\}}$, $[u, u]^{\{n\}},[X, u]^{a v g},[u, u]^{a v g}$, and respective covariances. These can be found in the technical appendix, Kalnina and Linton (2007). From there, $2 \bar{n}^{1 / 2}[X, u]^{a v g}-\frac{\bar{n}^{3 / 2}}{n} 2[X, u]^{\{n\}}=o_{p}(1)$ by Chebyshev's inequality and similarly $\frac{\bar{n}^{3 / 2}}{n}[X, X]^{\{n\}}=o_{p}(1)$. Also, we have $\mathrm{E}\left([X, X]^{\text {avg }}-Q V_{X}\right)=o\left(\bar{n}^{-1 / 2}\right)$ from ZMA (2005) and $E\left[\bar{n}^{1 / 2}[u, u]^{\text {avg }}-\frac{\bar{n}^{3 / 2}}{n}[u, u]^{\{n\}}\right]=o(1)$. Therefore,

$$
\begin{aligned}
& \bar{n}^{1 / 2}\left(\widehat{Q V}_{X}-Q V_{X}\right) \\
= & \bar{n}^{1 / 2}\left([X, X]^{a v g}-\mathrm{E}[X, X]^{a v g}+[u, u]^{a v g}-E[u, u]^{a v g}-\frac{1}{K}[u, u]^{\{n\}}+\frac{1}{K} E[u, u]^{\{n\}}\right)+o_{p}(1) .
\end{aligned}
$$


We use Berk's (1973) central limit theorem for $m$-dependent variables with $m=1$. Note that we can prove the CLT for the special case $\alpha=0$ and convergence rate $n^{1 / 6}$, then get the needed result by multiplying and dividing the main expression by $n^{\alpha / 3}$. We proceed in the case where all three terms contribute, which is the case where $K$ is chosen optimally to be $K=O\left(n^{2 / 3}\right)$. Also, we can do all calculations, conditional on $\sigma=\left\{\sigma_{t}, t \in[0,1]\right\}$. Then, since $\sigma$ is independent of all other randomness, we can conclude the same CLT unconditionally. We apply Berk's CLT to the following sums of $U_{n i}$,

$$
\begin{aligned}
T_{n}= & V(\sigma)^{-1} \bar{n}^{1 / 2}\left([X, X]^{a v g}-\mathrm{E}[X, X]^{a v g}+[u, u]^{a v g}-E[u, u]^{a v g}-\frac{1}{K}[u, u]^{[n\}}+\frac{1}{K} E[u, u]^{\{n\}}\right) \\
= & \bar{n}^{-1 / 2} \sum_{i=1}^{\bar{n}-1} V(\sigma)^{-1 / 2} U_{n i}, \\
U_{n i}= & \frac{\bar{n}}{K}\left\{\sum_{j=1}^{K}\left(X_{t_{i K+j}}-X_{t_{(i-1) K+j}}\right)^{2}-\int_{t_{(i-1) K+j}}^{t_{i K+j}} \sigma_{u}^{2} d u\right\} \\
& +\frac{\bar{n}}{K}\left\{\sum_{j=1}^{K}\left(u_{t_{i K+j}}-u_{t_{(i-1) K+j}}\right)^{2}-E\left(u_{t_{i K+j}}-u_{t_{(i-1) K+j}}\right)^{2}\right\} \\
& -\frac{\bar{n}}{K}\left\{\sum_{s=1}^{2 K-1} \frac{1}{2}\left(u_{(i-1) K+1+s}-u_{(i-1) K+s}\right)^{2}-E \frac{1}{2}\left(u_{(i-1) K+1+s}-u_{(i-1) K+s}\right)^{2}\right\} \\
\equiv & U_{n i}^{x}+U_{n i}^{u_{1}}+U_{n i}^{u_{2}} \equiv U_{n i}^{x}+U_{n i}^{u} .
\end{aligned}
$$

There are 4 conditions to be satisfied in Berk's CLT, which we denote (i)-(iv). Notice that $\left\{U_{n i}\right\}_{i=1}^{\bar{n}-1}$ is (conditionally on $\sigma$ ) a sequence of 1-dependent random variables. Therefore, condition (iv) on dependence is trivially satisfied. Condition (iii) requires the following to exist and be non-zero,

$$
V(\sigma)=\lim _{n \rightarrow \infty} \bar{n}^{-1} \operatorname{var}\left\{\sum_{i=1}^{\bar{n}-1} U_{n i}\right\} .
$$

This follows by our moment calculations,

$$
\begin{aligned}
V(\sigma)= & \lim _{n \rightarrow \infty}\left\{\bar{n} \operatorname{var}[X, X]^{a v g}+\bar{n} \operatorname{var}[u, u]^{a v g}+\left(\frac{\bar{n}^{3 / 2}}{n}\right)^{2} \operatorname{var}[u, u]^{\{n\}}-2 \frac{\bar{n}^{2}}{n} \operatorname{cov}\left([u, u]^{[n\}},[u, u]^{a v g}\right)\right\} \\
= & \frac{4}{3} \int \sigma_{t}^{4} d t+\frac{2}{c^{3}}\left(12 \delta^{4}+4 E \epsilon^{4} \int \omega^{4}(u) d u+24 \delta^{2} \int \omega^{2}(u) d u\right) \\
& -\frac{2}{c^{3}}\left(8 \delta^{4}+4\left(E \epsilon^{4}-1\right) \int \omega^{4}(u) d u+16 \delta^{2} \int \omega^{2}(u) d u\right) \\
= & \frac{4}{3} \int \sigma_{t}^{4} d t+c^{-3}\left(8 \delta^{4}+16 \delta^{2} \int \omega^{2}(u) d u+8 \int \omega^{4}(u) d u\right) .
\end{aligned}
$$


Condition (ii) requires

$$
\operatorname{var}\left(U_{n s+1}+\ldots+U_{n s^{\prime}}\right) \leq\left(s^{\prime}-s\right) M^{\prime} \text { for all } i, j \text {, and } n \text { sufficiently large, }
$$

where $M^{\prime}$ is some constant. We have that

$$
\begin{aligned}
\operatorname{var}\left(U_{n s+1}^{x}+\ldots+U_{n s^{\prime}}^{x}\right)= & \operatorname{var}\left(\bar{n} \frac{1}{K} \sum_{j=1}^{K} \sum_{i=s+1}^{s^{\prime}}\left\{\left(X_{t_{i K+j}}-X_{t_{(i-1) K+j}}\right)^{2}-\int_{t_{(i-1) K+j}}^{t_{i K+j}} \sigma_{u}^{2} d u\right\}\right) \\
\leq & 2\left(s^{\prime}-s\right)\left\{\sup _{u \in[0,1]} \sigma^{2}(u)\right\} \\
\operatorname{var}\left(U_{n s+1}^{u}+\ldots+U_{n s^{\prime}}^{u}\right)= & \frac{\bar{n}^{2}}{K^{2}} \sum_{i=s+1}^{s^{\prime}}\left\{4 \sum_{j=1}^{K} \operatorname{var}\left(u_{i K+j} u_{(i-1) K+j}\right)+\sum_{j=1}^{2 K-1} \operatorname{var}\left(u_{(i-1) K+1+j} u_{(i-1) K+j}\right)\right\} \\
& +\frac{\bar{n}^{2}}{K^{2}} \sum_{i=s+1}^{s^{\prime}}\left\{\frac{1}{4} \operatorname{var}\left(u_{i K-K+1}^{2}\right)-\frac{3}{4} \operatorname{var}\left(u_{i K+K}^{2}\right)+\operatorname{var}\left(u_{i K+1}^{2}\right)\right\}+o(1) \\
\leq & \left(s^{\prime}-s\right) C_{u}\left\{6 c^{-3}+c^{-4}\right\}+o(1),
\end{aligned}
$$

where the $o(1)$ terms arise from the mean $m($.$) and are asymptotically negligible, while c$ is the constant in the definition of $K$ and $C_{u}$ is the maximum of the upper bound for $\left(\operatorname{var}\left(u_{i}\right)\right)^{2}$ and the upper bound for $\operatorname{var}\left(u_{i}^{2}\right)$. Their respective expressions are as follows:

$$
\begin{aligned}
\operatorname{var}\left(u_{i}\right) \leq & \delta^{2}+\left\{\sup _{t \in[0,1]} \omega(t)\right\}^{2} \\
\operatorname{var}\left(u_{i}^{2}\right) \leq & 2 \delta^{4}+4\left\{\sup _{t \in[0,1]} m(t)\right\}^{2}\left\{\sup _{t \in[0,1]} \omega(t)\right\}^{2}+4 \sup _{t \in[0,1]} m(t)\left\{\sup _{t \in[0,1]} \omega(t)\right\}^{3} \mathrm{E}|\epsilon|^{3} \\
& +\left\{\sup _{t \in[0,1]} \omega(t)\right\}^{4}\left(\mathrm{E} \epsilon^{4}-1\right)+4\left\{\sup _{t \in[0,1]} m(t)\right\}^{2} \delta^{2}+4\left\{\sup _{t \in[0,1]} \omega(t)\right\}^{2} \delta^{2} .
\end{aligned}
$$

By the Cauchy-Schwarz inequality we obtain (13).

Finally, condition (i) is:

For some $\eta>0$ and $M<\infty, \mathrm{E}\left|U_{n i}\right|^{2+\eta} \leq M$ for all $i$ and $n$.

Then for some constant $C, E\left[\left|U_{n i}\right|^{2+\eta}\right] \leq C\left(E\left[\left|U_{n i}^{x}\right|^{2+\eta}\right]+E\left[\left|U_{n i}^{u_{1}}\right|^{2+\eta}\right]+E\left[\left|U_{n i}^{u_{2}}\right|^{2+\eta}\right]\right)$. Different arguments are required for the $U_{n i}^{x}$ and $U_{n i}^{u}$ terms - the summands in $U_{n i}^{x}$ are highly dependent but individually of small order while the summands in $U_{n i}^{u}$ are independent or of low order dependence but of individually larger order. Define

$$
w_{n i j}=\frac{n}{K}\left[\left(X_{t_{i K+j}}-X_{t_{(i-1) K+j}}\right)^{2}-\int_{t_{(i-1) K+j}}^{t_{i K+j}} \sigma_{u}^{2} d u\right] .
$$


Then, since $X_{t_{i K+j}}-X_{t_{(i-1) K+j}} \sim N\left(0, \int_{t_{(i-1) K+j}}^{t_{i K+j}} \sigma_{u}^{2} d u\right)$, where $\int_{t_{(i-1) K+j}}^{t_{i K+j}} \sigma_{u}^{2} d u=O(K / n)$, we have $E\left[\left|w_{n i j}\right|^{r}\right] \leq C_{r}<\infty$ for all $r, i, j$. Note that $X_{t_{i K+j}}-X_{t_{(i-1) K+j}}$ and $X_{t_{i K+j^{\prime}}}-X_{t_{(i-1) K+j^{\prime}}}$ for $j \neq j^{\prime}$ are highly dependent. We write

$$
U_{n i}^{x}=\frac{\bar{n}}{K}\left\{\sum_{j=1}^{K}\left(X_{t_{i K+j}}-X_{t_{(i-1) K+j}}\right)^{2}-\int_{t_{(i-1) K+j}}^{t_{i K+j}} \sigma_{u}^{2} d u\right\}=\frac{\bar{n}}{K} \frac{K}{n} \sum_{j=1}^{K} w_{n i j} .
$$

Therefore, by Minkowski inequality

$$
\left(E\left[\left|U_{n i}^{x}\right|^{2+\eta}\right]\right)^{1 / 2+\eta} \leq \frac{\bar{n} K}{K n} \sum_{j=1}^{K}\left(E\left[\left|w_{n i j}\right|^{2+\eta}\right]\right)^{1 / 2+\eta}=\frac{1}{K} \sum_{j=1}^{K}\left(E\left[\left|w_{n i j}\right|^{2+\eta}\right]\right)^{1 / 2+\eta}<\infty .
$$

Similar arguments apply to the terms $U_{n i}^{u_{1}}$ and $U_{n i}^{u_{2}}$, where we make use of the assumption that $E\left[\left|\epsilon_{t_{i}}\right|^{4+\eta}\right]<\infty$. We just show the argument for $U_{n i}^{u_{1}}$. Recall that $u_{t_{i K+j}}-u_{t_{(i-1) K+j}}=v_{t_{i K+j}}-v_{t_{(i-1) K+j}}+$ $\varepsilon_{t_{i K+j}}-\varepsilon_{t_{(i-1) K+j}}$, where $v_{t_{i}}=\delta \sqrt{n}\left(W_{t_{i}}-W_{t_{i-1}}\right)$ and $\varepsilon_{t_{i}}=m\left(t_{i}\right)+\omega\left(t_{i}\right) \epsilon_{t_{i}}$, so it suffices to show this result for the two components. The arguments to do with $v_{t_{i}}$ are straightforward because all moments exist and the magnitude is just right. Regarding the $\varepsilon_{t_{i K+j}}$ terms, let $\xi_{n j}=\left(\varepsilon_{t_{i K+j}}-\varepsilon_{t_{(i-1) K+j}}\right)^{2}-$ $E\left(\varepsilon_{t_{i K+j}}-\varepsilon_{t_{(i-1) K+j}}\right)^{2}$, where $\xi_{n j}$ are independent and mean zero random variables across $j=1, \ldots, K$. First, notice that $E\left[\left|\epsilon_{t_{i K+j}}\right|^{4+2 \eta]}<\infty, \sup _{u \in[0,1]}|m(u)|<\infty\right.$, and $\sup _{u \in[0,1]}|\omega(u)|<\infty$ imply that $E\left[\left|\varepsilon_{t_{i K+j}}\right|^{4+2 \eta}\right]<\infty$. Then, $E\left[\left|\varepsilon_{t_{i K+j}}\right|^{4+2 \eta}\right]<\infty$ implies that $E\left[\left|\left(\varepsilon_{t_{i K+j}}-\varepsilon_{t_{(i-1) K+j}}\right)^{2}\right|^{2+\eta}\right]<\infty$ and hence $E\left[\left|\xi_{n j}\right|^{2+\eta}\right]$. Then, by the Marcinkiewicz-Zygmund inequality for independent random variables and Hölder's inequality for sums

$$
\begin{aligned}
E\left[\left|\sum_{j=1}^{K} \xi_{n j}\right|^{p}\right] & \leq C_{p} E\left[\left|\sum_{j=1}^{K} \xi_{n j}^{2}\right|^{p / 2}\right] \\
& \leq C_{p} E\left[\left|\left(\sum_{j=1}^{K}\left|\xi_{n j}\right|^{p}\right)^{2 / p}\right|^{p / 2}\right] \\
& =C_{p}\left(\sum_{j=1}^{K} E\left|\xi_{n j}\right|^{p}\right)<\infty
\end{aligned}
$$

for any $p$ for which $E\left|\xi_{n j}\right|^{p}<\infty$. It follows that

$$
E\left[\left|U_{n i}^{\varepsilon}\right|^{2+\eta}\right] \equiv E\left[\left|\frac{\bar{n}}{K} \sum_{j=1}^{K} \xi_{n j}\right|^{2+\eta}\right] \leq\left(\frac{\bar{n}}{K}\right)^{2+\eta} K C_{2+\eta}<\infty
$$

for $K=O\left(n^{2 / 3}\right)$. This establishes condition (i). 
To conclude, the conditions of Berk's theorem are satisfied conditional on $\sigma$ and so we have shown that $\operatorname{Pr}\left(T_{n} \leq t \mid \sigma\right) \rightarrow \Phi(t)$ for all $t$, which implies that $\operatorname{Pr}\left(T_{n} \leq t\right) \rightarrow \Phi(t)$, where $\Phi(t)$ denotes the c.d.f. of a standard normal random variable.

\section{References}

[1] Andersen, T. G. and T. Bollerslev (1997). Intraday periodicity and volatility persistence in financial markets. Journal of Empirical Finance 5, 115 - 158.

[2] Andersen, T. G., T. Bollerslev, F. X. Diebold, and P. Labys. (2000), Great realizations, Risk 13(3), 105-108.

[3] Andersen, T. G., T. Bollerslev, and N. Meddahi (2006). Realized Volatility Forecasting and Market Microstructure Noise. Working Paper.

[4] Aït-Sahalia, Y., and L. Mancini (2006). Out-of-sample forecasts of Quadratic Variation. Working Paper.

[5] Aït-Sahalia, Y., P. Mykland, and L. Zhang (2005). How Often to Sample a ContinuousTime Process in the Presence of Market Microstructure Noise. Review of Financial Studies, 18, $351-416$.

[6] Aït-Sahalia, Y., P. Mykland, and L. Zhang (2006a). Ultra high frequency volatility estimation with dependent microstructure noise. Unpublished paper: Department of economics, Princeton University

[7] Aït-Sahalia, Y., P. Mykland, and L. Zhang (2006b). Comments on 'Realized Variance and Market Microstructure Noise,' by P. Hansen and A. Lunde, Journal of Business and Economic Statistics.

[8] Awartani, B., Corradi, V., and W. Distaso (2004). Testing and Modelling Market Microstructure Effects with and Application to the Dow Jones Industrial Average. Working paper.

[9] Back, K. And S. Baruch (2004). Kyle meets Glosten and Milgrom. Econometrica 72(2), 433-465.

[10] Bandi, F. M., And J. R. Russell (2006a). Market microstructure noise, integrated variance estimators, and the accuracy of asymptotic approximations. Working paper, Chicago GSB. 
[11] Bandi, F. M., And J. R. Russell (2006b). Separating microstructure noise from volatility. Journal of Financial Economics 79, 655-692.

[12] Bandi, F. M., And J. R. Russell (2006c). Comment on "Realized Variance and Microstructure Noise" by Peter Hansen and Asger Lunde. Journal of Business and Economic Statistics, $24,167-173$

[13] Bandi, F. M., J. R. Russell, and C. Yang (2007). Realised Volatility Forecasting and Option Pricing. Working paper, Chicago GSB.

[14] Barndorff-Nielsen, O. E., P. R. Hansen, A. Lunde, and N. Shephard (2006). Designing Realised Kernels to Measure the Ex-post Variation of Equity Prices in the Presence of Noise. Working Paper.

[15] Barndorff-Nielsen, O. E., And N. Shephard (2002). Econometric analysis of realised volatility and its use in estimating stochastic volatility models. Journal of the Royal Statistical Society B 64, 253-280.

[16] Barndorff-Nielsen, O. E., and N. Shephard (2007). Variation, jumps, market frictions and high frequency data in financial econometrics. Advances in Economics and Econometrics. Theory and Applications, Ninth World Congress, (edited by Richard Blundell, Persson Torsten and Whitney K Newey), Econometric Society Monographs, Cambridge University Press.

[17] Berk, K. N. (1973). A Central Limit Theorem for m-dependent Random Variables with Unbounded m. The Annals of Probability, Vol. 1, No. 2, 352-354.

[18] Bound, J., C. Brown, And N. Mathiowetz (2001). Measurement error in survey data. In the Handbook of Econometrics, Eds. J.J. Heckman and E. Leamer vol. 5. 3705-3843.

[19] Copeland, T. E., and D. Galai (1983). Information Effects on the Bid-Ask Spread. The Journal of Finance, Vol. 38, No. 5, 1457-1469.

[20] Dahlhaus, R. (1997). Fitting time series models to nonstationary processes. Annals of Statistics $25,1-37$.

[21] Diebold, F.X. (2006). On Market Microstructure Noise and Realized Volatility. Discussion of Hansen and Lunde (2006).

[22] Gerety, M. S., And H. Mulherin (1994). Price formation on stock exchanges: The evolution of trading within the day, Review of Financial Studies 6, 23-56. 
[23] Ghysels, E. And A. Sinko (2006). Volatility Forecasting and Microstructure Noise. Working paper, University of North Carolina.

[24] Gonçalves, S., And N. Meddahi (2005). Bootstrapping Realized Volatility. Unpublished paper.

[25] Gloter, A., And J.Jacod (2001). Diffusions with measurement errors. I - Local asymptotic normality. ESAIM: Probability and Statistics 5, 225-242.

[26] Gottlieb, G., And A. Kalay (1985). Implications of the Discreteness of Observed Stock Prices. The Journal of Finance XL, 135-153.

[27] Hall, P., And C. C. Heyde (1980). Martingale Limit Theory and Its Applications. Academic Press, New York

[28] Hansen, P. R., And A. Lunde (2006). Realized variance and market microstructure noise (with comments and rejoinder). Journal of Business and Economic Statistics 24, 127-218.

[29] Heston, S. (1993). A Closed-Form Solution for Options With Stochastic Volatility With Applications to Bonds and Currency Options. Review of Financial Studies, 6, 327-343.

[30] HARRIS, L. (1986). A transaction data study of weekly and intradaily patterns in stock returns. Journal of Financial Economics 16, 99-117.

[31] Jacod, J., And P. Protter (1998). Asymptotic error distribution for the Euler method for stochastic differential equations. Annals of Probability 26, 267-307.

[32] Kalnina, I. And Linton, O. B., (2007) Technical appendix. http://personal.lse.ac.uk/KALNINA/tech_app.pdf

[33] Kleidon, A., And I. Werner (1996). U.K. and U.S. trading of British cross-listed stocks: An intraday analysis of market integration. Review of Financial Studies 9, 619-644.

[34] Kristensen, D. (2006). Filtering of the realised volatility: a kernel-based approach. Working paper, University of Wisconsin.

[35] Large, G. (2007). Estimating Quadratic Variation When Quoted Prices Change by a Constant Increment. Nuffield College Economics Group Working Paper.

[36] Li, Y., And P. A. Mykland (2006). Determining the Volatility of a Price Process in the presence of rounding errors. Working Paper, Department of Statistics, the University of Chicago. 
[37] Li, Y., And P. A. Mykland (2007). Are volatility estimators robust with respect to modelling assumptions? Bernoulli 13 (3), 601-622.

[38] Linton, O. B., And I. Kalnina (2007). Discussion of Aït-Sahalia and Shephard. World Congress of the Econometric Society. Forthcoming Cambridge University Press

[39] Lockwood, L. J., AND S. C. Linn (1990). An examination of stock market return volatility during overnight and intraday periods, 1964-1989. Journal of Finance 45, 591-601.

[40] McInish, T. H., And R. A. Wood (1992). An analysis of intraday patterns in bid/ask spreads for NYSE stocks. Journal of Finance 47, 753-764.

[41] Maddala, G.S. (1977). Econometrics. McGraw Hill, New York.

[42] Robinson, P.M. (1986). On the errors in variables problem for time series. Journal of Multivariate Analysis 19, 240-250.

[43] Rosenbaum, M. (2007). Integrated Volatility and round-off error. Working paper, Université PARIS-EST and CREST-ENSAE.

[44] ZhANG, L. (2004). Efficient estimation of stochastic volatility using noisy observations: a multiscale approach. Bernoulli. Forthcoming.

[45] Zhang, L., P. Mykland, and Y. Aït-Sahalia (2005a). A tale of two time scales: determining integrated volatility with noisy high-frequency data. Journal of the American Statistical Association, 100, 1394-1411

[46] Zhang, L., P. Mykland, and Y. AïT-Sahalia (2005b). Edgeworth Expansions for Realized Volatility and Related Estimators. Working paper, Princeton University.

[47] Zhou, B. (1996). High-frequency data and volatility in foreign-exchange rates. Journal of Business and Economic Statistics 14, 45-52. 


\section{B Tables and Figures}

Table 2. IQR percentage error with $K=\left(2 V_{2} / V_{1}\right)^{1 / 3} n^{\frac{2}{3}(1-\alpha)}$

\begin{tabular}{c|cccccccccccc}
\hline \hline$n \backslash \alpha$ & 0 & 0.05 & 0.1 & 0.15 & 0.2 & 0.25 & 0.3 & 0.35 & 0.4 & 0.45 & 0.5 \\
\hline 195 & 96 & 186 & 145 & 120 & 145 & 114 & 95 & 78 & 65 & 54 & N/A \\
390 & 94 & 135 & 110 & 200 & 156 & 128 & 143 & 111 & 89 & 71 & 59 \\
780 & 67 & 90 & 108 & 137 & 107 & 181 & 151 & 162 & 119 & 100 & 76 \\
1560 & 55 & 74 & 67 & 86 & 94 & 125 & 205 & 161 & 119 & 125 & 92 \\
4680 & 48 & 47 & 56 & 58 & 74 & 96 & 99 & 117 & 201 & 144 & 151 \\
5850 & 44 & 51 & 57 & 57 & 66 & 81 & 76 & 135 & 98 & 160 & 163 \\
7800 & 45 & 46 & 52 & 53 & 68 & 70 & 90 & 94 & 109 & 175 & 134 \\
11700 & 40 & 44 & 45 & 52 & 53 & 59 & 81 & 78 & 141 & 208 & 148 \\
23400 & 36 & 40 & 43 & 46 & 49 & 58 & 61 & 79 & 106 & 123 & 196 \\
\hline \hline & & & Table 3. & $K$ & $\left(2 V_{2} / V_{1}\right)^{1 / 3} n^{\frac{2}{3}(1-\alpha)}$ & & & \\
\hline \hline$n \backslash \alpha$ & 0 & 0.05 & 0.1 & 0.15 & 0.2 & 0.25 & 0.3 & 0.35 & 0.4 & 0.45 & 0.5 \\
\hline 195 & 3 & 2 & 2 & 2 & 1 & 1 & 1 & 1 & 1 & 1 & 0 \\
390 & 4 & 3 & 3 & 2 & 2 & 2 & 1 & 1 & 1 & 1 & 1 \\
780 & 7 & 5 & 4 & 3 & 3 & 2 & 2 & 1 & 1 & 1 & 1 \\
1560 & 11 & 8 & 7 & 5 & 4 & 3 & 2 & 2 & 2 & 1 & 1 \\
4680 & 22 & 17 & 13 & 10 & 7 & 5 & 4 & 3 & 2 & 2 & 1 \\
5850 & 26 & 19 & 14 & 11 & 8 & 6 & 5 & 3 & 3 & 2 & 1 \\
7800 & 31 & 23 & 17 & 13 & 9 & 7 & 5 & 4 & 3 & 2 & 2 \\
11700 & 41 & 30 & 22 & 16 & 12 & 9 & 6 & 5 & 3 & 2 & 2 \\
23400 & 65 & 47 & 33 & 24 & 17 & 12 & 9 & 6 & 4 & 3 & 2 \\
\hline \hline
\end{tabular}


Table 4. IQR percentage error with $K=n^{\frac{2}{3}(1-\alpha)}$

\begin{tabular}{c|ccccccccccc}
\hline \hline$\backslash \alpha$ & 0 & 0.05 & 0.1 & 0.15 & 0.2 & 0.25 & 0.3 & 0.35 & 0.4 & 0.45 & 0.5 \\
\hline 195 & -21 & -16 & -13 & -7 & -7 & -3 & -1 & 4 & 8 & 13 & 13 \\
390 & -15 & -12 & -7 & -3 & -3 & 1 & 3 & 6 & 7 & 12 & 14 \\
780 & -13 & -11 & -4 & -2 & 0 & 0 & 4 & 5 & 6 & 11 & 14 \\
1560 & -9 & -7 & -2 & -1 & 1 & 3 & 5 & 7 & 8 & 13 & 12 \\
4680 & -5 & -3 & -1 & -2 & 1 & 0 & 3 & 5 & 6 & 7 & 11 \\
5850 & -4 & -3 & 1 & 3 & 5 & 5 & 2 & 4 & 8 & 8 & 8 \\
7800 & -2 & -2 & 0 & 1 & 3 & 2 & 5 & 3 & 6 & 8 & 10 \\
11700 & -3 & 0 & 0 & 2 & 2 & 5 & 4 & 2 & 6 & 3 & 8 \\
23400 & -2 & 1 & 2 & 1 & 3 & 4 & 2 & 6 & 6 & 6 & 8 \\
\hline \hline
\end{tabular}

Table $5 . K=n^{\frac{2}{3}(1-\alpha)}$

\begin{tabular}{c|ccccccccccc}
\hline \hline$n \backslash \alpha$ & 0 & 0.05 & 0.1 & 0.15 & 0.2 & 0.25 & 0.3 & 0.35 & 0.4 & 0.45 & 0.5 \\
\hline 195 & 34 & 28 & 24 & 20 & 17 & 14 & 12 & 10 & 8 & 7 & 6 \\
390 & 53 & 44 & 36 & 29 & 24 & 20 & 16 & 13 & 11 & 9 & 7 \\
780 & 85 & 68 & 54 & 44 & 35 & 28 & 22 & 18 & 14 & 11 & 9 \\
1560 & 135 & 105 & 82 & 64 & 50 & 39 & 31 & 24 & 19 & 15 & 12 \\
4680 & 280 & 211 & 159 & 120 & 91 & 68 & 52 & 39 & 29 & 22 & 17 \\
5850 & 325 & 243 & 182 & 136 & 102 & 76 & 57 & 43 & 32 & 24 & 18 \\
7800 & 393 & 292 & 216 & 161 & 119 & 88 & 66 & 49 & 36 & 27 & 20 \\
11700 & 515 & 377 & 276 & 202 & 148 & 108 & 79 & 58 & 42 & 31 & 23 \\
23400 & 818 & 585 & 418 & 299 & 214 & 153 & 109 & 78 & 56 & 40 & 29 \\
\hline \hline
\end{tabular}

Table 6. IQR percentage error with $K=n^{\frac{2}{3}}$

\begin{tabular}{c|ccccccccccc|c}
\hline \hline$\backslash \alpha$ & 0 & 0.05 & 0.1 & 0.15 & 0.2 & 0.25 & 0.3 & 0.35 & 0.4 & 0.45 & 0.5 & $K$ \\
\hline 195 & -23 & -23 & -24 & -23 & -23 & -21 & -23 & -24 & -23 & -24 & -23 & 34 \\
390 & -17 & -19 & -19 & -17 & -19 & -20 & -18 & -16 & -16 & -18 & -18 & 53 \\
780 & -14 & -15 & -12 & -15 & -14 & -12 & -15 & -15 & -16 & -14 & -13 & 85 \\
1560 & -12 & -9 & -10 & -10 & -12 & -11 & -11 & -9 & -11 & -12 & -9 & 135 \\
4680 & -7 & -2 & -7 & -5 & -5 & -7 & -6 & -5 & -5 & -6 & -5 & 280 \\
5850 & -6 & -6 & -6 & -6 & -6 & -6 & -5 & -7 & -6 & -5 & -4 & 325 \\
7800 & -5 & -6 & -4 & -4 & -3 & -4 & -5 & -4 & -5 & -6 & -5 & 393 \\
11700 & -2 & -6 & -3 & -3 & -3 & -4 & -2 & -5 & -6 & -2 & -3 & 515 \\
23400 & -2 & -2 & -3 & -2 & -1 & -2 & -1 & -3 & -4 & -2 & -4 & 818 \\
\hline \hline
\end{tabular}


Table 7. IQR percentage error with $K^{B R}=\phi=\left(\frac{3 R V^{2}}{2 R Q}\right)^{1 / 3} n^{1 / 3}$

\begin{tabular}{c|ccccccccccc|c}
\hline \hline$n \backslash \alpha$ & 0 & 0.05 & 0.1 & 0.15 & 0.2 & 0.25 & 0.3 & 0.35 & 0.4 & 0.45 & 0.5 & $K^{B R}$ \\
\hline 195 & 55 & 46 & 34 & 29 & 27 & 21 & 22 & 19 & 16 & 18 & 15 & 6 \\
390 & 67 & 49 & 37 & 28 & 23 & 20 & 17 & 18 & 15 & 15 & 14 & 8 \\
780 & 94 & 65 & 48 & 32 & 26 & 22 & 19 & 16 & 16 & 14 & 12 & 10 \\
1560 & 124 & 81 & 54 & 36 & 27 & 24 & 15 & 14 & 14 & 13 & 13 & 13 \\
4680 & 243 & 146 & 91 & 54 & 34 & 24 & 18 & 16 & 12 & 14 & 8 & 18 \\
5850 & 263 & 155 & 92 & 53 & 35 & 24 & 18 & 11 & 11 & 11 & 12 & 20 \\
7800 & 300 & 182 & 97 & 60 & 33 & 26 & 15 & 13 & 10 & 11 & 9 & 22 \\
11700 & 381 & 223 & 125 & 68 & 39 & 24 & 17 & 11 & 12 & 9 & 8 & 25 \\
23400 & 539 & 305 & 163 & 86 & 47 & 28 & 15 & 13 & 8 & 8 & 8 & 32 \\
\hline \hline
\end{tabular}

Table 8. Effect of $\delta^{2}$ on the estimates $\widehat{Q V}_{x}$

\begin{tabular}{c|c|c}
\hline \hline$\delta^{2} / \delta_{b}^{2}$ & $\operatorname{corr}\left(\Delta X_{t_{i}}, \Delta u_{t_{i}}\right)$ & IQR error \\
\hline 0.01 & -0.0010 & 0.0133 \\
0.05 & -0.0051 & 0.0128 \\
0.1 & -0.0102 & 0.0049 \\
0.25 & -0.0254 & 0.0182 \\
0.5 & -0.0506 & 0.0037 \\
1 & -0.1000 & 0.0136 \\
2 & -0.1909 & 0.0100 \\
4 & -0.3280 & 0.0090 \\
10 & -0.4869 & 0.0130 \\
20 & -0.5351 & 0.0105 \\
\hline \hline
\end{tabular}




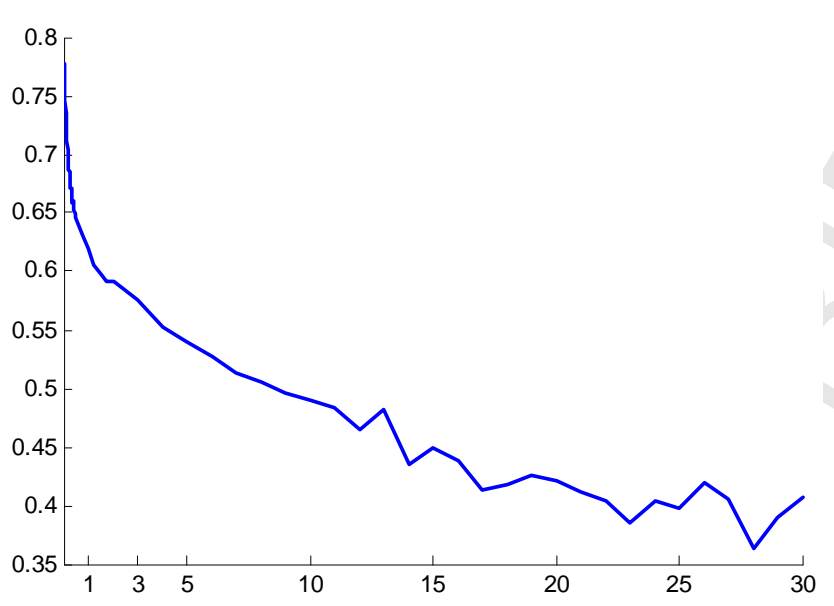

Figure 5. Volatility signature plot for IBM transactions data, year 2005. The scale on the X axis is the frequency of interpolated calendar time observations, in minutes. Y axis denotes average of daily RV using calendar time data at frequency specified by the $\mathrm{x}$ axis. 